\title{
The Eddington factor as the key to understand the winds of the most massive stars
}

\section{Evidence for a Г-dependence of Wolf-Rayet type mass loss}

\author{
G. Gräfener ${ }^{1}$, J. S. Vink ${ }^{1}$, A. de Koter $^{2,4}$, and N. Langer ${ }^{3,4}$
}

\author{
1 Armagh Observatory, College Hill, Armagh BT61 9DG, UK \\ 2 Astronomical Institute "Anton Pannekoek", University of Amsterdam, Science Park XH, Amsterdam, The Netherlands \\ 3 Argelander-Institut für Astronomie der Universität Bonn, Auf dem Hügel 71, 53121 Bonn, Germany \\ 4 Astronomical Institute, Utrecht University, Princetonplein 5, 3584 CC Utrecht, The Netherlands
}

Received 11 February 2011 / Accepted 30 June 2011

\begin{abstract}
Context. The most massive stars are thought to be hydrogen-rich Wolf-Rayet stars of late spectral subtype (in the following WNh stars). The emission-line spectra of these stars are indicative of strong mass loss. In previous theoretical studies this enhanced mass loss has been attributed to their proximity to the Eddington limit.

Aims. We investigate observed trends in the mass-loss properties of such young, very massive stars to examine a potential $\Gamma$-dependence, i.e., with respect to the classical Eddington factor $\Gamma_{\mathrm{e}}$. Based on different mass estimates, we gain information about the evolutionary status of these objects.

Methods. We derive theoretical mass-luminosity relations for very massive stars, based on a large grid of stellar structure models. Using these relations, we estimate Eddington factors $\left(\Gamma_{\mathrm{e}}\right)$ for a sample of stars, under different assumptions of their evolutionary status. We evaluate the resulting mass-loss relations, and compare them with theoretical predictions.

Results. We find observational evidence that the mass loss in the WR regime is dominated by the Eddington parameter $\Gamma_{\mathrm{e}}$, which has important consequences for the way we understand Wolf-Rayet stars and their mass loss. In addition, we derive wind masses that support the picture that the WNh stars in young stellar clusters are very massive, hydrogen-burning stars.

Conclusions. Our findings suggest that the proximity to the Eddington limit is the physical reason for the onset of Wolf-Rayet type mass loss. This means that, e.g. in stellar evolution models, the Wolf-Rayet stage should be identified by large Eddington parameters, instead of a helium-enriched surface composition. The latter is most likely only a consequence of strong mass loss, in combination with internal mixing. For very massive stars, the enhanced $\Gamma$-dependent mass loss is responsible for the formation of late WNh subtypes with high hydrogen surface abundances, partly close to solar. Because mass loss dominates the evolution of very massive stars, we expect a strong impact of this effect on their end products, in particular on the potential formation of black holes, and gamma-ray bursts, as well as the observed upper mass limit of stars.
\end{abstract}

Key words. stars: Wolf-Rayet - stars: early-type - stars: atmospheres - stars: mass-loss - stars: winds, outflows

\section{Introduction}

Wolf-Rayet (WR) type mass loss fundamentally affects the evolution, the final fate, and the chemical yields of massive stars. The amount of mass loss in the WR phase predominantly decides whether a star ends its life as neutron star, or black hole (Heger et al. 2003). In particular, WR-type mass loss at low metallicities $(Z)$ is expected to be of paramount importance for the chemical enrichment of the early universe (Meynet et al. 2006; Chiappini et al. 2006), and the formation of long-duration gamma-ray bursts (long GRBs, Yoon \& Langer 2005; Woosley \& Heger 2006).

The nature of WR-type mass loss is however still poorly understood. Stellar evolution models mostly rely on empirical mass-loss relations (e.g. Nugis \& Lamers 2000; Hamann et al. 2006), with the WR phase identified on the basis of observed WR surface abundances in our galaxy. Clearly, for the modeling of stellar populations that cannot be observed locally, a more physical approach would be desirable.

In the present work we elaborate on such an approach, namely a mass-loss relation for WR stars that chiefly depends on the Eddington factor $\Gamma_{\mathrm{e}}$ (Eq. (1)). Such relations have been predicted for very massive stars close to the Eddington limit (Gräfener \& Hamann 2008; Vink et al. 2011), and for LBVs (Vink \& de Koter 2002). Notably, the proximity to the Eddington limit provides a natural explanation for the occurrence of the WR phenomenon. Large Eddington factors can be reached, on the one hand, by very massive stars on the main sequence because of their extremely high luminosities, and on the other hand, by less massive evolved (He-burning) stars due to the enhanced mean molecular weight in their cores. The occurrence of WRtype mass loss for young, luminous, hydrogen-rich stars (typically late WNh subtypes), and evolved, hydrogen-free WR stars (nitrogen-rich WN, and carbon-rich WC subtypes) can thus be explained in the same way.

The main goal of this paper is to confront the theoretically predicted concept of $\Gamma$-dependent mass loss with the observed mass-loss properties of very massive stars. We use the results of a study of the most massive stars in the Arches cluster near the Galactic centre (GC), by Martins et al. (2008). The Arches cluster is a young star forming region rich in very massive stars, 
including many $\mathrm{O}$ and $\mathrm{Of}+$ supergiants, and luminous WNh stars. It forms an ideal testbed for our present study.

Our paper is organized in the following way. In Sect. 2 we briefly describe the properties of luminous WNh stars. Section 3 recaps our theoretical knowledge of the mass-loss properties of these objects. To study the dependence of mass loss on $\Gamma_{\mathrm{e}}$ requires mass estimates for the sample stars. In Sect. 4 we provide mass versus luminosity relations for chemically-homogeneous stars, that may be used to derive the Eddington factor for the Arches stars from their observed luminosities, and surface abundances. In Sect. 5, we study the dependence of the mass-loss rates on stellar properties, including $\Gamma_{\mathrm{e}}$, and compare these dependencies with theoretical predictions. The main intention in this section is to perform a qualitative comparison. At the present time a quantitative comparison may be affected by systematic uncertainties. Our findings are discussed in Sect. 6, and conclusions are drawn in Sect. 7.

\section{Physical properties of the most massive stars}

The most massive stars with direct mass estimates are found in binary systems that contain luminous, hydrogen-rich WNh stars. The highest masses lie in the range of 70-120 $M_{\odot}$ (Rauw et al. 1996; Schweickhardt et al. 1999; Rauw et al. 2004; Bonanos et al. 2004; Schnurr et al. 2008, 2009). Furthermore, spectral analyses of (putatively) single WNh stars imply very high masses. Typical luminosities lie in the range of $10^{6} L_{\odot}$ or higher, implying that most WNh stars are very massive stars in the phase of core H-burning (Crowther et al. 1995; Crowther \& Smith 1997; Crowther \& Dessart 1998; de Koter et al. 1997; Hamann et al. 2006; Martins et al. 2008, 2009; Crowther et al. 2010).

Luminous WNh stars are preferentially found in the centers of massive, young stellar clusters with ages of only a few Myr. This, combined with the fact that their surfaces still show ample amounts of hydrogen, suggests that they are still in their phase of core H-burning. Well-known examples are the Arches cluster close to the GC, the young galactic cluster NGC 3603, and $\mathrm{R} 136$, the central cluster of the star-forming region 30 Dor in the Large Magellanic Cloud (LMC). Crowther et al. (2010) recently determined a luminosity of $10^{6.94} L_{\odot}$ for the brightest star in R 136, which would correspond to a single star with a presentday mass of $265 M_{\odot}$.

The fact that the most massive stars appear as Wolf-Rayet spectral types can be explained as a result of their proximity to the Eddington limit. According to Gräfener \& Hamann (2008), the increased density scale height close to the Eddington limit leads to the formation of strong winds with large optical depths. Because ionizing photons are efficiently absorbed within these winds, recombination sets in. This shows up in the form of strong WR emission lines that originate from the subsequent recombination cascades.

In the following we characterize the proximity to the Eddington limit by the "classical" Eddington factor

$\Gamma_{\mathrm{e}}=\chi_{\mathrm{e}} L /(4 \pi c G M)$,

with the electron scattering opacity $\chi_{\mathrm{e}}{ }^{1}$. Because $\mathrm{H}$, and He are completely ionized in the inner regions of hot star atmospheres, $\Gamma_{\mathrm{e}}$ is nearly constant with radius, and depends only on the stellar parameters $M, L$, and, via the mass absorption coefficient $\chi_{\mathrm{e}}$, on the hydrogen mass fraction $X_{\mathrm{H}}^{\mathrm{s}}$ at the stellar surface (Eq. (8)).

\footnotetext{
${ }^{1}$ Note that the opacity $\chi$ is a mass absorption coefficient, i.e., in the CGS system it is measured in $\mathrm{cm}^{2} / \mathrm{g}$.
}

There exists a significant additional contribution to the total mean opacity $\chi(r)$, due to metal lines (chiefly Fe) and continua. For the physical, radius dependent Eddington factor $\Gamma(r)$, including all opacities, we thus have

$\Gamma(r)=\chi(r) L /(4 \pi c G M)>\Gamma_{\mathrm{e}}$.

The size of the shift between $\Gamma$ and $\Gamma_{\mathrm{e}}$ depends on the detailed metal abundances, and on the ionization structure of the atmosphere, and thus on $T_{\star}$, and Z. E.g., for solar metallicity models, Gräfener \& Hamann (2008) find that $\Gamma$ approaches unity in deep atmospheric layers, already for $\Gamma_{\mathrm{e}} \approx 0.5$. The onset of WR-type mass loss thus occurs for $\Gamma_{\mathrm{e}}<1$, i.e., for Eddington factors that are smaller, but still of the order of one.

\section{Mass-loss predictions for very massive stars}

Mass-loss predictions for very massive stars have been performed by Gräfener \& Hamann (2008), and Vink et al. (2011). Despite the rather different modeling approaches, these works agree on the dominant role of the Eddington factor $\Gamma_{\mathrm{e}}$ for the mass-loss properties of very massive stars.

The mass-loss relation by Gräfener \& Hamann (2008) is based on advanced stellar atmosphere models that incorporate non-LTE line blanketing, wind clumping, and an exact numerical solution of the hydrodynamic equations (Gräfener \& Hamann 2005). The models include complex model atoms of $\mathrm{H}, \mathrm{He}, \mathrm{C}$, $\mathrm{N}, \mathrm{O}, \mathrm{Si}$, and the Fe-group, which should be sufficient to describe the largest part of the radiative wind acceleration. We note however that the lack of intermediate elements $(\mathrm{Ne}-\mathrm{Ca})$ could potentially lead to an under-estimation of the mass-loss rates.

Apart from the dominant role of $\Gamma_{\mathrm{e}}$, Gräfener \& Hamann find a strong dependence on $T_{\star}$, and a strong $Z$-dependence. Their mass-loss prescription has the form

$$
\begin{aligned}
\log (\dot{M})= & -3.763 \\
& +\beta \log \left(\Gamma_{\mathrm{e}}-\Gamma_{0}\right)-3.5\left(\log \left(T_{\star} / \mathrm{K}\right)-4.65\right) \\
& +0.42\left(\log \left(L / L_{\odot}\right)-6.3\right)-0.45\left(X_{\mathrm{H}}-0.4\right),
\end{aligned}
$$

with $\dot{M}$ in $M_{\odot} \mathrm{yr}^{-1}$, and the $Z$-dependent parameters $\beta$, and $\Gamma_{0}$ given by

$$
\begin{aligned}
\beta(Z) & =1.727+0.250 \log \left(Z / Z_{\odot}\right), \\
\Gamma_{0}(Z) & =0.326-0.301 \log \left(Z / Z_{\odot}\right)-0.045 \log \left(Z / Z_{\odot}\right)^{2} .
\end{aligned}
$$

Note that the stellar temperature $T_{\star}$ denotes the effective core temperature as defined, e.g., in Gräfener et al. (2002).

Vink et al. (2011) compute mass-loss rates for very massive stars using a Monte Carlo approach with a parameterized solution of the hydrodynamic equations (Müller \& Vink 2008). They confirm the dominant role of $\Gamma_{\mathrm{e}}$, but find a weak temperature dependence in the range of 30-50 kK. Notably, Vink et al. resolve the transition between classical OB star mass loss, with a relatively weak dependence on $\Gamma_{\mathrm{e}}$ (Eq. (6)), and WR-type mass loss with a much steeper dependence (Eq. (7)). However, Vink et al. note that the precise value of $\Gamma_{\mathrm{e}}$ where this transition occurs in their models, might be too high. The likely reason is that the shift between the Eddington factor $\Gamma(r)$, and $\Gamma_{\mathrm{e}}$ (cf. Eq. (2)) is underestimated in their models (see also the discussion in Sect. 6.2).

For a solar composition, and an effective temperature of $50 \mathrm{kK}$ Vink et al. give relations of the form

$$
\begin{array}{ll}
\log (\dot{M}) \propto 1.52 \log \left(\Gamma_{\mathrm{e}}\right)+0.68 \log \left(L / L_{\odot}\right) & \Gamma_{\mathrm{e}}<0.7 \\
\log (\dot{M}) \propto 3.99 \log \left(\Gamma_{\mathrm{e}}\right)+0.78 \log \left(L / L_{\odot}\right) & \Gamma_{\mathrm{e}}>0.7, \\
\text { with } \dot{M} \text { in } M_{\odot} \mathrm{yr}^{-1} . &
\end{array}
$$


For the Eddington factor $\Gamma_{\mathrm{e}}$ both works adopt the value for a fully ionized plasma, which is given by

$$
\log \left(\Gamma_{\mathrm{e}}\right)=-4.813+\log \left(1+X_{\mathrm{H}}^{\mathrm{s}}\right)+\log \left(L / L_{\odot}\right)-\log \left(M / M_{\odot}\right) .
$$

In this form $\Gamma_{\mathrm{e}}$ only depends on the stellar parameters $M, L$, and, via $\chi_{\mathrm{e}}$, on the hydrogen mass fraction $X_{\mathrm{H}}^{\mathrm{s}}$ at the stellar surface (cf. Eq. (1)).

The strong sensitivity to the Eddington factor in both massloss relations ${ }^{2}$ offers the potential to provide very precise estimates of $\Gamma_{\mathrm{e}}$ for specific objects. If $\dot{M}, L, X_{\mathrm{H}}^{\mathrm{s}}$, and $Z$ are known from spectral analyses, it is thus possible to obtain very precise mass estimates, within the systematic errors of the adopted massloss relation.

A major goal of the present work is to calibrate the underlying mass-loss relation by a comparison of such "wind masses" with predicted masses from stellar structure computations. If the important dependencies on $\Gamma_{\mathrm{e}}, T_{\star}$, and $Z$ are backed up by observations, the mass-loss relations may not only serve as input for stellar evolution computations, but can also provide an important diagnostic tool to examine the present masses, and thus the evolutionary status of observed stars.

\section{Mass-luminosity relations for very massive stars}

In the present section, we provide theoretical $M-L$ relations for very massive stars. These relations can be used to estimate masses $M$, and Eddington factors $\Gamma_{\mathrm{e}}$ for observed stars with known stellar parameters $L, T_{\star}$, and $X_{\mathrm{H}}^{\mathrm{s}}$.

A basic problem of such an approach is that the internal structure of a single observed star is generally not known, i.e., its precise mass cannot be uniquely predicted. In the present section we thus focus on the extremes, namely the lowest and highest masses for a star with given observed parameters. Applying these relations to a large sample of stars, we will be able to perform a qualitative investigation of their mass-loss properties, and to examine patterns that are related to the stellar core, and surface abundances.

The highest possible mass for a star with given luminosity $L$, and hydrogen surface abundance $X_{\mathrm{H}}^{\mathrm{s}}$ is reached by chemicallyhomogeneous stars. Under this assumption, the star is characterized by one, constant hydrogen abundance $X_{\mathrm{H}}$, which equals the surface abundance $X_{\mathrm{H}}^{\mathrm{s}}$. The estimated stellar mass $M_{\mathrm{hom}}\left(L, X_{\mathrm{H}}^{\mathrm{s}}\right)$ is strongly dependent on the (observable) surface abundance $X_{\mathrm{H}}^{\mathrm{s}}$. Stars that are not homogeneous have a higher mean molecular weight in the core than at the surface. They thus have higher $L / M$ ratios, or lower masses, for given $L$, and $X_{\mathrm{H}}^{\mathrm{s}}$.

The lowest possible mass is given by the completely inhomogeneous case, i.e., by a core hydrogen abundance $X_{\mathrm{H}}^{\mathrm{c}}=0$. In this case the star is in the core He-burning phase ${ }^{3}$. Lauterborn et al. (1971) have investigated how luminosities, and temperatures of core He-burning stars vary, depending on the mass ratio between the H-rich envelope and the He core. According to this work, there exists a generalized main sequence that depends on the size of the He core. Stars with very small $\mathrm{He}$ cores display hot temperatures, and similar luminosities as their H-burning counterparts. For larger cores, temperatures become very cool, and luminosities increase with increasing core size.

\footnotetext{
2 The steepness of the $\Gamma_{\mathrm{e}}$-dependence in Eq. (3) is mainly due to the fact that $\log \left(\Gamma_{\mathrm{e}}-\Gamma_{0}\right)$ is evaluated for $\Gamma_{\mathrm{e}}-\Gamma_{0}$ close to zero.

3 Note that we only focus on stars in the phase of central $\mathrm{H}$, or $\mathrm{He}$ burning because the burning timescales in later phases become extremely short.
}

Finally, stars with large He cores show the same luminosities as pure He-stars, and hot temperatures. Because we are interested in hot, massive stars with large convective cores in this work, we can thus use the $M-L$ relation for pure He-stars for the core He-burning case. Our minimum mass is thus given by $M_{\mathrm{Heb}}(L) \equiv M_{\text {hom }}\left(L, X_{\mathrm{H}}=0\right)$. Note that this mass estimate is completely independent of the (observed) surface abundance $X_{\mathrm{H}}^{\mathrm{s}}$.

The different dependence on $X_{\mathrm{H}}^{\mathrm{s}}$ in both cases is of paramount importance for the present work. Because of the expected relation between mass and mass loss, we will be able to identify patterns in the observed properties of very massive stars that help to distinguish between samples of well-mixed stars, that are quasi-chemically homogeneous, and stars with a pronounced chemical profile.

\subsection{The $M-L$ relation for homogeneous stars}

In the present section we derive analytical expressions of the form $L\left(M, X_{\mathrm{H}}\right)$, and $M\left(L, X_{\mathrm{H}}\right)$, for the masses and luminosities of chemically-homogeneous stars. As discussed above, we expect the luminosity $L$ to depend on the mass $M$, and the hydrogen mass fraction $X_{\mathrm{H}}$ for core H-burning stars, and only on $M$ for core He-burning stars. To investigate this dependence we have computed a grid of homogeneous stellar structure models for the mass range $M=0.3-4000 M_{\odot}$, and hydrogen mass fractions $X_{\mathrm{H}}=0.0-0.7$, at solar metallicity.

The stellar structure models are computed with a simple code that integrates the stellar structure equations using a shooting method. The numerical methods are described in the textbook by Hansen \& Kawaler (1994). The code is based on an example program that is distributed with the book, but is completely re-written, and updated with OPAL opacities (Iglesias \& Rogers 1996). To circumvent numerical problems with the extended envelopes of extremely massive stars (see Ishii et al. 1999; Petrovic et al. 2006), we have adopted an outer boundary temperature that lies above the temperature of the Fe-opacity peak ( $160 \mathrm{kK})$. In this way we start our computations just below the extended envelopes. Because the masses of such envelopes are very small $\left(<10^{-2} M_{\star}\right)$ this approach has no effect on the obtained luminosities.

The results of our grid computations are displayed in Figs. 1 and 2. They are in good agreement with previous models by Langer (1989), and Ishii et al. (1999) that comprise a much smaller parameter range. We find that a polynomial relation that is quadratic in $\log (M)$, and linear in $X_{\mathrm{H}}$ fits the results satisfactorily over the parameter range $M=12-250 M_{\odot}$, and $X_{\mathrm{H}}=0.1-0.7$ (see Figs. 1 and 2). The resulting relation has the form

$$
\begin{aligned}
\log \left(L / L_{\odot}\right) & =\left[F_{1}+F_{2} X_{\mathrm{H}}\right] \\
& +\left[F_{3}+F_{4} X_{\mathrm{H}}\right] \log \left(M / M_{\odot}\right) \\
& +\left[F_{5}+F_{6} X_{\mathrm{H}}\right] \log \left(M / M_{\odot}\right)^{2},
\end{aligned}
$$

with the coefficients $F_{1}-F_{6}$ from row No. 1 in Table A.1. For $X_{\mathrm{H}}<0.1$, the changes in the core temperature become so large that the dependence on $X_{\mathrm{H}}$ becomes significantly non-linear. We thus restrict our fit to $X_{\mathrm{H}}>0.1$, and derive a separate relation for pure He stars $\left(X_{\mathrm{H}}=0\right)$, over a mass range of $8-250 M_{\odot}$. This relation has the form

$\log \left(L / L_{\odot}\right)=F_{1}+F_{2} \log \left(M / M_{\odot}\right)+F_{3} \log \left(M / M_{\odot}\right)^{2}$,

where the coefficients $F_{1}-F_{3}$ are again given in row No. 6 in Table A.1. The maximum fitting error for $\log \left(L / L_{\odot}\right)$ over the 


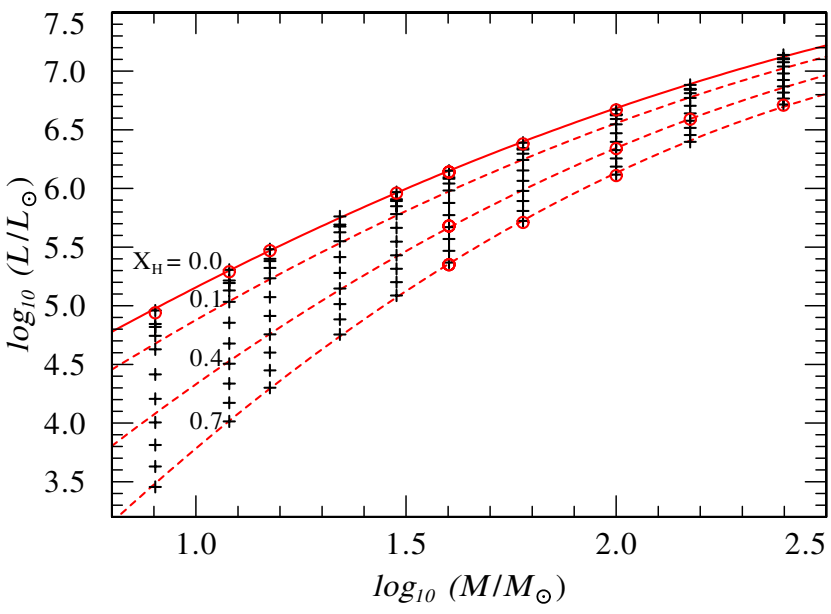

Fig. 1. Homogeneous stellar structure models for the mass range 8$250 M_{\odot}$ (black symbols). The models are computed for hydrogen mass fractions $X_{\mathrm{H}}=0.7-0$ (from bottom to top). Dashed red lines indicate the $M-L$ relations according to Eq. (9) and row No. 1 in Table A.1 for $X_{\mathrm{H}}=0.7,0.4$, and 0.1 , and the solid red line corresponds to pure $\mathrm{He}$ models according to Eq. (10) and row No. 6 in Table A.1. For comparison, model computations from Ishii et al. (1999) are indicated by red circles. The fitting relations are inferred for the mass ranges of 12$250 M_{\odot}$ (Eq. (9)), and 8-250 $M_{\odot}$ (Eq. (10)).

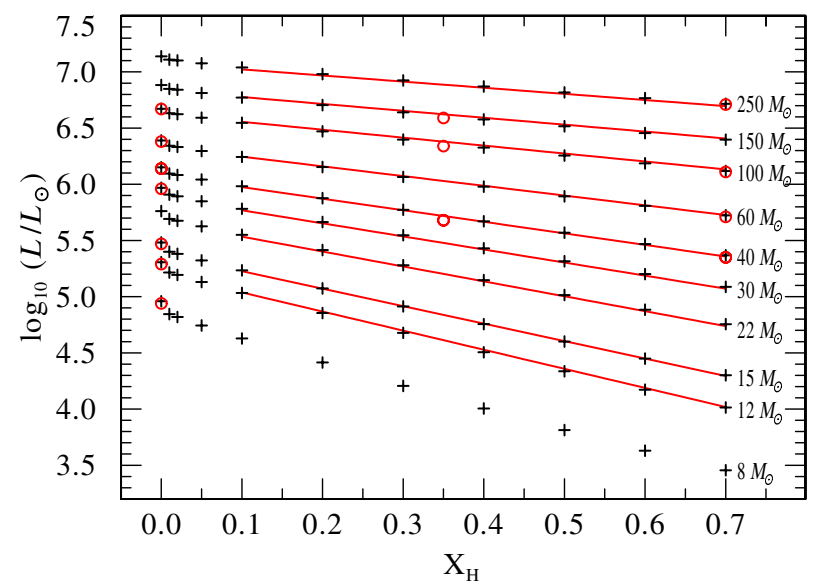

Fig. 2. $M-L$ relation for chemically-homogeneous stars, dependence on $X_{\mathrm{H}}$. The symbols are the same as in Fig. 1. Red lines indicate our linear fit to models with constant mass but varying hydrogen mass fraction $X_{\mathrm{H}}$, for a mass range of $12-250 M_{\odot}$, according to Eq. (9) and row No. 1 in Table A.1.

given parameter ranges amounts to 0.02 . Inverting these relations we obtain the masses $M_{\mathrm{hom}}\left(L, X_{\mathrm{H}}\right)$, and $M_{\mathrm{Heb}}(L)$ in the form

$\log \left(M_{\mathrm{hom}} / M_{\odot}\right)=\frac{F_{1}+F_{2} X_{\mathrm{H}}+F_{3} \sqrt{f}}{1+F_{9} X_{\mathrm{H}}}$

with

$f=F_{4}+F_{5} X_{\mathrm{H}}+F_{6} X_{\mathrm{H}}^{2}+\left(F_{7}+F_{8} X_{\mathrm{H}}\right) \log \left(L / L_{\odot}\right)$,

and

$\log \left(M_{\mathrm{Heb}} / M_{\odot}\right)=F_{1}+F_{2} \sqrt{F_{3}+F_{4} \log \left(L / L_{\odot}\right)}$.

All coefficients are given in Table A.1, together with coefficients for additional relations that cover higher, and lower mass ranges. The latter are discussed in Appendix A, and may be useful for future studies.

\section{The most massive stars in the Arches cluster}

In the present section we confront the concept of $\Gamma$-dependent mass-loss rates with observations. To this end we use the large, and well studied sample of very massive stars in the Arches cluster, near the GC. The core of this young massive cluster contains 13 extremely luminous WNh stars, and a similar amount of bright early-type $\mathrm{O}$, and Of stars. We adopt the stellar parameters $L, X_{\mathrm{H}}^{\mathrm{s}}, T_{\star}$, and $\dot{M}$, as obtained by Martins et al. $(2008)^{4}$ in a comprehensive study of the Arches sample.

In Sect. 5.1 we start with a description of the properties of the Arches cluster stars. Using a fitting technique, we investigate in Sect. 5.2, whether the observed properties of the Arches stars are in line with a general $\Gamma$-dependent mass loss relation. In Sect. 5.3 we determine wind masses from the mass-loss relations by Gräfener \& Hamann (2008), to test if the theoretical relations cover a realistic parameter range.

\subsection{Properties of the most massive stars in the Arches cluster}

The stellar parameters of the Arches stars, as derived by Martins et al. (2008), are compiled in Table 1. Spectral types reach from early $\mathrm{O}$, and Of supergiants to late WNh subtypes. Stellar temperatures lie in the range $T_{\star}=30-40 \mathrm{kK}$, and luminosities in the range $\log \left(L_{\star} / L_{\odot}\right)=5.75-6.35$, with the WNh stars showing systematically higher luminosities, and lower temperatures. Moreover, the large part of the WNh stars is H-deficient and Nenriched, with respect to solar values. Note, however, that some WNh stars display a solar hydrogen abundance at their surface.

Based on the derived mass-loss rates, Martins et al. (2008) identify two distinct wind momentum - luminosity relations for the O/Of, and WNh stars. The WNh stars display systematically higher mass-loss rates than the $\mathrm{O}$ stars. This raises the question in which way the WNh stars differ from the O/Of stars. On the one hand, the different mass-loss properties could indicate a different evolutionary stage, e.g., the WNh stars could be in the phase of core He-burning. On the other hand, parameters like surface abundances, or effective temperatures could be responsible for the observed dichotomy.

\section{2. $\Gamma$-dependent mass-loss rates}

In this section we investigate whether the observed properties of the Arches cluster stars are in line with a $\Gamma$-dependent mass-loss relation. For this purpose we adopt a general mass loss relation in the form of a power law with four free parameters

$$
\begin{aligned}
\log \left(\dot{M} / M_{\odot} \mathrm{yr}^{-1}\right)=\dot{M}_{0}+f_{\Gamma} \log \left(\Gamma_{\mathrm{e}}\right) \\
\quad+f_{L}\left(\log \left(L / L_{\odot}\right)-6.0\right)+f_{T}\left(\log \left(T_{\star} / \mathrm{K}\right)-4.5\right)
\end{aligned}
$$

To estimate $\Gamma_{\mathrm{e}}$, we use Eq. (8), with the relations for $M_{\mathrm{hom}}\left(L, X_{\mathrm{H}}\right)$, and $M_{\mathrm{Heb}}(L)$ from Sect. 4.1. This way, it is possible to constrain the free parameters $\dot{M}_{0}, f_{\Gamma}, f_{L}$, and $f_{T}$ by a $\chi^{2}$-fit, based on the observed values of $\dot{M}, L, T_{\star}$, and $X_{\mathrm{H}}^{\mathrm{s}}$.

${ }^{4}$ Note that the effective core temperature $T_{\star}$, given by Martins et al. (2008), is defined in the same way as by Gräfener \& Hamann (2008), as the effective temperature related to the inner boundary radius $R_{\star}$ of the model atmosphere, i.e., by the relation $L=4 \pi R_{\star}^{2} \sigma T_{\star}^{4}$, where $R_{\star}$ is located at large optical depth. Due to the small density scale height in these layers, the precise value of the reference optical depth has almost no influence on the value of $T_{\star}$, so that the temperatures given by Martins et al. are ideally suited for the use with the relation by Gräfener \& Hamann. 
Table 1. Stellar parameters of the most massive stars in the Arches cluster.

\begin{tabular}{|c|c|c|c|c|c|c|c|c|c|c|c|c|c|c|c|}
\hline Star & Subtype & $\begin{array}{l}T_{\star} \\
{[\mathrm{kK}]}\end{array}$ & $\begin{array}{l}L \\
{\left[L_{\odot}\right]}\end{array}$ & $\begin{array}{l}\log (\dot{M}) \\
{\left[\frac{M_{\odot}}{\mathrm{yr}}\right]} \\
\end{array}$ & $\begin{array}{l}v_{\infty} \\
{\left[\mathrm{km} \mathrm{s}^{-1}\right]}\end{array}$ & $X_{\mathrm{H}}^{\mathrm{s}}$ & $\begin{array}{l}X_{\mathrm{N}}^{\mathrm{s}} \\
{[\%]}\end{array}$ & $\begin{array}{l}M_{\mathrm{Heb}} \\
{\left[M_{\odot}\right]} \\
\end{array}$ & $\begin{array}{l}M_{\mathrm{hom}} \\
{\left[M_{\odot}\right]}\end{array}$ & $\begin{array}{l}M_{\mathrm{w} 2} \\
{\left[M_{\odot}\right]}\end{array}$ & $\begin{array}{l}M_{\mathrm{w} 1} \\
{\left[M_{\odot}\right]}\end{array}$ & $\Gamma_{\mathrm{e}}^{\mathrm{Heb}}$ & $\Gamma_{\mathrm{e}}^{\mathrm{hom}}$ & $\Gamma_{\mathrm{e}}^{\mathrm{w} 1}$ & $\Gamma_{\mathrm{e}}^{\mathrm{w} 2}$ \\
\hline F8 & WN8-9 & 33.7 & 6.10 & -4.50 & 1000 & 0.18 & 1.64 & 37.1 & 55.5 & 51.0 & 43.3 & 0.63 & 0.42 & 0.46 & .54 \\
\hline F5 & WN8-9 & & 5 & -4.64 & 900 & 0.22 & & 29.5 & 47.3 & 36 & 21 & 0.58 & 0.36 & 0.46 & 0.54 \\
\hline F3 & WN8-9 & 29.9 & 6.10 & -4.60 & 800 & 0.27 & 2.79 & 37.1 & 62.7 & & & 0.68 & 0.40 & .40 & .48 \\
\hline F4 & WN7-8 & & & -4.35 & 1400 & & & & & & & & 0.45 & 0.56 & 0.64 \\
\hline F2 & WN8-9 & & & -4.72 & 1400 & 0.40 & & & & & & & & 0.44 & 0.52 \\
\hline F7 & WN8-9 & & & & 1300 & & & & 10 & & & & & 44 & 52 \\
\hline F6 & WN8-9 & & & - & 1400 & & & & 12 & & & 7 & & 15 & .53 \\
\hline F12 & $\mathrm{WN}$ & & & 4.7 & 0 & & 2. & 43 & & & & & & 6 & .54 \\
\hline B1 & WN8- & & & -5.00 & 1600 & 0.6 & 2.41 & 29 & 82 & 4 & & 0. & & 9 & .47 \\
\hline F1 & WN8-9 & 3.7 & .30 & -4.70 & 1400 & 0.6 & 1.45 & 51. & 13 & 11 & 10 & 1.03 & 0 & 0.44 & 0.52 \\
\hline F9 & WN8-9 & 36.8 & 6.35 & -4.78 & 1800 & 0.69 & 1.46 & 55. & 143.6 & 131.3 & 111.3 & 1.07 & 0.41 & 0.45 & 0.53 \\
\hline F14 & WN8-9 & 1.5 & 6.00 & -5.00 & 1400 & 0.69 & 0.49 & 31. & 87.4 & 64 & 54.0 & 0.83 & 0.30 & 0.41 & 0.49 \\
\hline F16 & WN8-9 & .4 & 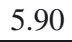 & -5.11 & 1400 & 0.69 & 1.46 & 27 & 76 & 56 & 45 & 0.76 & 0.27 & 0.37 & 0.46 \\
\hline F10 & GIf & & 5.9 & 53 & 0 & 0 & 0 & & & & & 0. & & 34 & .42 \\
\hline F15 & $\mathrm{O}$ & & & & 00 & & & & & & & 3 & & 8 & .47 \\
\hline F18 & $\mathrm{O} 4$ & & & - & 0 & 9 & 0 & 3 & 3 & 82. & 6 & 6 & & 36 & .44 \\
\hline $\mathrm{F} 20$ & $\mathrm{O}_{2}$ & 3.4 & & - & & 0 & 0 & 27 & 7 & 57. & 4 & 0. & 7 & 6 & .45 \\
\hline $\mathrm{F} 21$ & O4-6I & .8 & & -5.49 & 2200 & 0.69 & 0. & 29 & 82 & 70 & 56 & 0.80 & 0 . & 0.33 & 0.42 \\
\hline F22 & O4-6I & 5.8 & ) & -5.70 & 1900 & 0.69 & 0.39 & 23.7 & 68.0 & 52 & 41. & 0.70 & 0.24 & 0.32 & 0.40 \\
\hline F23 & O4-6I & 5.8 & 80 & -5.65 & 00 & 0.69 & 0.69 & 23.7 & 68.0 & 51 & 40.7 & 0.70 & 0.24 & 0.32 & 0.41 \\
\hline F26 & O4-6I & & & -5.73 & 00 & 0.6 & 0.40 & 25. & 72. & 56 & 45.0 & 0.73 & 0.26 & 0.33 & 0.42 \\
\hline F28 & O4-6 & & & -5.70 & & & & & & & & 0. & & 0.33 & 0.41 \\
\hline F29 & $\mathrm{O}_{2}$ & & & -5.6 & 2900 & 0.69 & 0.30 & 22.1 & 64.1 & 44.8 & 35.6 & 0.67 & 0.23 & 0.33 & 0.42 \\
\hline F32 & O4-6I & 40.8 & 5.8 & -5.90 & 2400 & 0.69 & 0.29 & 25.5 & 72.3 & 59.3 & 46.6 & 0.73 & 0.26 & 0.31 & 0.40 \\
\hline F33 & O4-6I & 39.8 & 5.85 & -5.73 & 2600 & 0.69 & 0.39 & 25.5 & 72.3 & 56.6 & 45.0 & 0.73 & 0.26 & 0.33 & 0.42 \\
\hline F34 & O4-6I & 38.1 & 5.75 & -5.77 & 1750 & 0.69 & 0.40 & 22.1 & 64.1 & 46.0 & 36.3 & 0.67 & 0.23 & 0.32 & 0.40 \\
\hline F35 & O4-6 & 33.8 & 5.70 & -5.76 & 2150 & 0.69 & 0.20 & 20.6 & 60.5 & 43.1 & 33.6 & 0.64 & 0.22 & 0.31 & 0.39 \\
\hline F40 & O4-6I & 39.8 & 5.75 & -5.75 & 2450 & 0.69 & 0.40 & 22.1 & 64.1 & 44.5 & 35.5 & 0.67 & 0.23 & 0.33 & 0.42 \\
\hline
\end{tabular}

Notes. Designations, subtypes, stellar temperatures $\left(T_{\star}\right)$, luminosities $(L)$, mass-loss rates $(\dot{M})$, terminal wind velocities $\left(v_{\infty}\right)$, hydrogen, and nitrogen surface mass fractions $\left(X_{\mathrm{H}}^{\mathrm{s}}, X_{\mathrm{N}}^{\mathrm{s}}\right)$, from Martins et al. (2008), and mass estimates from the present work: He-burning masses $\left(M_{\mathrm{Heb}}\right)$, and homogeneous masses $\left(M_{\text {hom }}\right)$ according to Eqs. (11-13), with coefficients from rows 11, and 16 in Table A.1, wind masses according to Gräfener \& Hamann (2008) for $Z=2 Z_{\odot}\left(M_{\mathrm{w} 2}\right)$, and $Z=1 Z_{\odot}\left(M_{\mathrm{w} 1}\right)$. The corresponding Eddington factors $\Gamma_{\mathrm{e}}$ are given in the last four columns.

The hydrogen surface abundance $X_{\mathrm{H}}^{\mathrm{s}}$ plays a crucial role in this fitting process, as it enters the estimate of $\Gamma_{\mathrm{e}}$ via $\chi_{\mathrm{e}}$ (Eq. (8)), and via $M_{\text {hom }}\left(L, X_{\mathrm{H}}\right)$. The wide spread in surface abundances for the WN stars in our sample is important for the verification of an actual $\Gamma$-dependence. Without variations in $X_{\mathrm{H}}^{\mathrm{s}}$, a $\Gamma$-dependence could not be easily distinguished from an $L$-dependence, if there exists an underlying $M(L)$ relation that does not depend on other parameters. In the following we will see that this may indeed happen for the $\mathrm{O}$ stars within our sample, which all have the same $X_{\mathrm{H}}^{\mathrm{s}}$.

Using Eq. (14), we perform $\chi^{2}$-fits to the observed mass-loss rates of the sample stars, based on the observed values of $L, T_{\star}$, and $X_{\mathrm{H}}^{\mathrm{s}}$. Dependent on whether $\Gamma_{\mathrm{e}}$ is estimated on the basis of $M_{\mathrm{Heb}}(L)$, or $M_{\mathrm{hom}}\left(L, X_{\mathrm{H}}\right)$, we designate the obtained values of $f_{\Gamma}$ in Eq. (14) as $f_{\Gamma}^{\text {hom }}$, or $f_{\Gamma}^{\text {Heb }}$. To extract the physically relevant parameters we perform a series of tests where the fitting parameters $f_{\Gamma}, f_{L}$, and $f_{T}$ are partly set to zero.

We start our investigation using only the $13 \mathrm{WNh}$ stars in the Arches sample. The results of our $\chi^{2}$-fits are compiled in Table 2. We start with the most simple case of a constant massloss rate, i.e., with $\dot{M}_{0}$ as the only free parameter. The obtained $\chi^{2}=0.544$ obviously marks the lower end of the achievable fit quality. In the next four rows of Table 2 we allow for one more free parameter apart from $\dot{M}_{0}$. Among those four cases the best result is obtained for a $\Gamma$-dependent mass-loss relation under the assumption of homogeneity. Note the big difference in $\chi^{2}$ between the homogeneous assumption, and the assumption of
Table 2. Results of various $\chi^{2}$ fits of Eq. (14) to the observed mass-loss rates of the $13 \mathrm{WNh}$ stars in the Arches sample.

\begin{tabular}{rrrrrr}
\hline \hline$\chi^{2}$ & $\dot{M}_{0}$ & \multicolumn{1}{c}{$f_{\Gamma}^{\text {hom }}$} & $f_{\Gamma}^{\text {Heb }}$ & $f_{L}$ & $f_{T}$ \\
\hline 0.544 & -4.721 & - & - & - & - \\
\hline 0.108 & -3.617 & 2.578 & - & - & - \\
0.536 & -4.752 & - & -0.330 & - & - \\
0.368 & -4.820 & - & - & 0.720 & - \\
0.531 & -4.751 & - & - & - & 1.204 \\
\hline 0.354 & -4.800 & - & - & 0.843 & -1.438 \\
0.039 & -2.802 & 4.208 & - & -0.845 & - \\
0.098 & -3.537 & 2.703 & - & - & -1.056 \\
0.037 & -5.244 & - & -2.937 & 1.749 & - \\
0.496 & -4.859 & - & -0.787 & - & 2.467 \\
\hline 0.037 & -2.768 & 4.293 & - & -0.920 & 0.495 \\
0.035 & -5.262 & - & -3.008 & 1.724 & 0.577 \\
\hline
\end{tabular}

Notes. Parameters indicated by (-) have been set to zero.

core He-burning. This is illustrated in Figs. 3 and 4, where the sample stars indeed form a well pronounced mass-loss relation under the assumption of homogeneity (Fig. 3), but not under the assumption of He-burning (Fig. 4). At this point we note that the two plots represent two extremes, and that the real $\dot{M}-\Gamma$ relation may lie in between the two cases. 


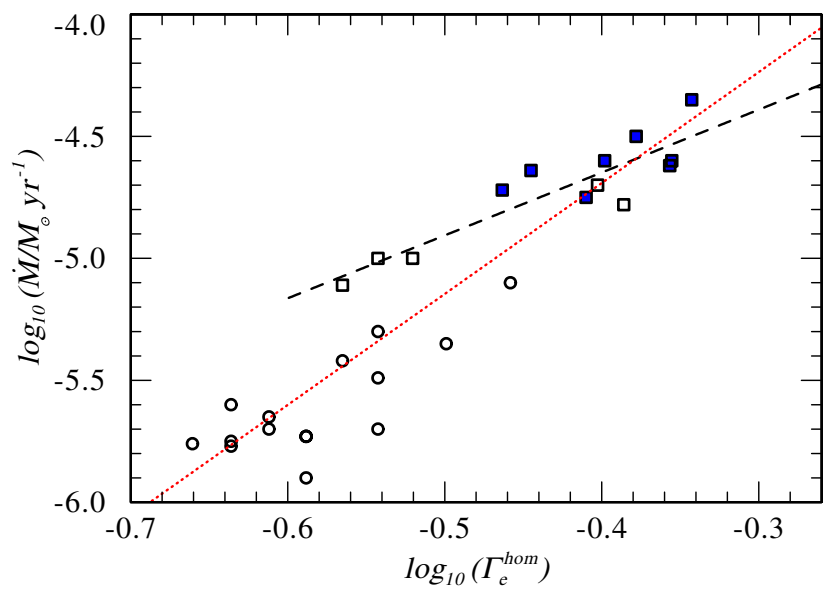

Fig. 3. Under the assumption of chemical homogeneity, the Arches cluster stars display a pronounced $\Gamma$-dependence. Squares indicate stars with spectral subtypes WNh (filled blue: $\mathrm{H}$-deficient; unfilled: normal $\mathrm{H}$ abundance), and circles O/Of. The black dashed line indicates the fitted mass-loss relation (Eq. (14)) for WNh stars (parameters from Table 2 row 2 ). The red dotted line indicates the corresponding fit for the complete sample (parameters from Table 3 row 2).

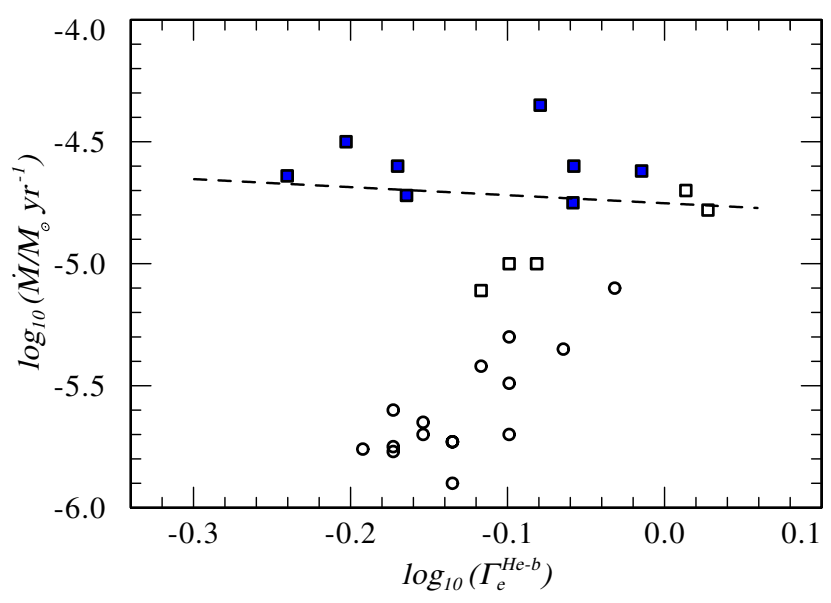

Fig. 4. Under the assumption of central He-burning, the Arches cluster stars display no general $\Gamma$-dependence. Symbols are the same as in Fig. 3, the black dashed line indicates a fitted relation (Eq. (14)) for WNh stars (parameters from Table 2 row 3 ).

The qualitative difference between the two plots originates from the fact that the adopted $M-L$ relation in Fig. 3 depends on $X_{\mathrm{H}}^{\mathrm{s}}$, while the relation in Fig. 4 does not. In both plots the $\mathrm{O}$ stars form a separate sequence that merges into the WR sequence. As explained above, this does not necessarily indicate a true $\Gamma$-dependence for the $\mathrm{O}$ star sample, as these stars show no variations in $X_{\mathrm{H}}^{\mathrm{s}}$. The WNh stars, on the other hand, display a substantial spread in $X_{\mathrm{H}}^{\mathrm{s}}$. Notably, in Fig. 4, the H-deficient stars show a significant shift towards lower $\Gamma_{\mathrm{e}}$, i.e., a "hook" in the $\dot{M}-\Gamma$ relation. This can be explained by the dependence of $\Gamma_{\mathrm{e}}$ on $X_{\mathrm{H}}^{\mathrm{s}}$ in Eq. (8), with $\Gamma_{\mathrm{e}} \propto 1+X_{\mathrm{H}}^{\mathrm{s}}$. The fact that this effect is precisely compensated in Fig. 3, suggests that the internal structure of the WNh stars in our sample is close to homogeneity. More precisely, it shows that there exists a relation between core, and surface hydrogen abundance. A similar effect may thus occur for inhomogeneous stars that have a similar degree of inhomogeneity, so that the differential behaviour of the homogeneous case is preserved. However, also in this case, the largest part of the
Table 3. Results of various $\chi^{2}$ fits of Eq. (14) to the observed mass-loss rates of the complete Arches sample.

\begin{tabular}{rrrrr}
\hline \hline$\chi^{2}$ & $\dot{M}_{0}$ & $f_{\Gamma}^{\text {hom }}$ & $f_{L}$ & $f_{T}$ \\
\hline 6.557 & -5.190 & - & - & - \\
\hline 0.942 & -2.871 & 4.550 & - & - \\
2.037 & -5.179 & - & 2.068 & - \\
4.160 & -4.880 & - & - & -8.458 \\
\hline 0.783 & -1.694 & 6.872 & -1.239 & - \\
0.457 & -2.957 & 3.997 & - & -4.116 \\
\hline 0.429 & -2.420 & 5.091 & -0.557 & -3.751 \\
\hline
\end{tabular}

Notes. Parameters indicated by $(-)$ have been set to zero.

sample stars need to be in phase of core H-burning to display the observed behaviour. Only few individual objects, with the highest He surface enrichment, may already have reached the He-burning phase.

In the remainder of Table 2 we allow for three or four free parameters. For these cases similar fitting results are obtained for the He-burning, and the homogeneous case. However, the obtained values for $f_{\Gamma}^{\text {Heb }}$ are all negative. Such a relation would imply that mass loss ceases close to the Eddington limit, which seems to be rather unphysical. An inspection of Fig. 4 shows that the assumption of He-burning would indeed imply a slightly negative slope of the mass-loss relation for WNh stars. The fact that for this case a similar fit quality can be achieved as for the homogeneous case is presumably a sign that the number of fitting parameters becomes too large for the small sample.

In Fig. 3 it is notable that our fitted $\dot{M}\left(\Gamma_{\mathrm{e}}\right)$ relation for WNh stars (black dashed line in Fig. 3) seems to be slightly detached from the rest of the sample, similar to the two different massloss relations for WN and O/Of stars identified by Martins et al. (2008). Figure 3, however, suggests that there might exist a smooth transition between the WNh, and O/Of stars (cf. Vink et al. 2011). To investigate this possibility we have performed the same fitting procedure as previously, for the complete Arches sample. Because the $\mathrm{O}$ stars are unlikely to be in the phase of core He-burning, we have however only focused on the homogeneous case.

The results are combined in Table 3. Again, we start with the simplest case of a constant mass-loss rate $\dot{M}_{0}$ with $\chi^{2}=6.557$. The next three rows allow for one more free parameter. Again, a $\Gamma$-dependent mass-loss relation is strongly favored with $\chi^{2}=$ 0.942. Interestingly, the following fits with more free parameters strongly favor a temperature dependence with $\dot{M} \propto T^{-4}$, which is very similar to the dependence predicted by Gräfener \& Hamann (2008). The two fits with the lowest $\chi^{2}$ in Table 3 do generally support the key features of their mass-loss predictions, which are 1) a strong $\Gamma$-dependence, 2 ) a weak luminosity dependence, and 3) a strong increase in mass loss for decreasing $T_{\star}$.

From row 6 in Table 3 we obtain the mass-loss relation

$$
\begin{aligned}
& \log \left(\dot{M} / M_{\odot} \mathrm{yr}^{-1}\right)= \\
& \quad-2.957+3.997 \log \left(\Gamma_{\mathrm{e}}\right)-4.116\left(\log \left(T_{\star} / \mathrm{K}\right)-4.5\right) .
\end{aligned}
$$

In Fig. 5 we compare this relation with observed mass-loss rates, compensating for the temperature dependence. Notably, the previous distinction between the $\mathrm{O} / \mathrm{Of}$, and the WNh sample from Fig. 3 does not exist anymore in this plot. The temperature difference between these two samples can thus account for the differences in their mass-loss rates. We note however that the 
G. Gräfener et al.: The Eddington factor as the key to understand the winds of the most massive stars

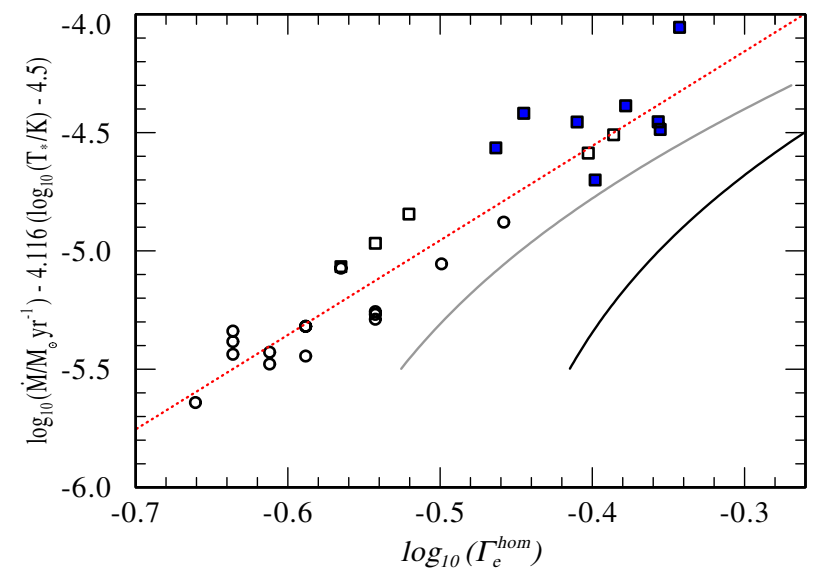

Fig. 5. Fitted mass-loss relation for the complete Arches sample, under the assumption of chemical homogeneity (Eq. (15), red dotted line). Symbols are the same as in Fig. 3. The observed mass-loss rates are scaled to a temperature of $\log \left(T_{\star} / \mathrm{K}\right)=4.5$, based on the temperature dependence in Eq. (15), with $\dot{M} \propto T^{-4.116}$. For comparison, we plot the relation by Gräfener \& Hamann (2008) for the same temperature, $\log (L)=6.3, X_{\mathrm{H}}=0.7$, and $Z=Z_{\odot}$ (black solid), as well as $Z=Z_{\odot}$ (grey solid).

dependence on $T_{\star}$ is uncertain, as it only follows from the combined $\mathrm{O}+\mathrm{WR}$ sample. From the WR sample alone there is no evidence for such a dependence, presumably due to the small spread in $T_{\star}$. At this time it is not clear at which point the transition to a $\Gamma$-dependent mass loss relation occurs, and to which extent such a relation is applicable to (parts of) the $\mathrm{O}$ star sample.

\subsection{Wind masses for the Arches cluster stars}

In the previous section we have shown that the observed properties of the Arches cluster stars are in line with a $\Gamma$-dependent mass-loss relation. The comparison in Fig. 5 shows that, under the assumption of chemical homogeneity, the resulting massloss relation is in good overall agreement with the relation by Gräfener \& Hamann (2008) for a high metallicity of $Z \sim 2 Z_{\odot}$. In the present section we use wind masses that follow from the relation by Gräfener \& Hamann, to investigate if the Arches stars conform with this mass-loss relation, and if the obtained wind masses cover a plausible range.

We derive wind masses by computing the Eddington factor $\Gamma_{\mathrm{e}}$ that is needed to explain the observed mass-loss rates $\dot{M}$, for given stellar parameters $L, T_{\star}, X_{\mathrm{H}}$, and $Z$, from Eqs. (3-5). The wind masses follow from $\Gamma_{\mathrm{e}}$, according to Eq. (8). In Table 1 we list the resulting masses, and Eddington factors. The different columns in Table 1 denote values obtained for an adopted metallicity of $2 Z_{\odot}\left(M_{\mathrm{w} 2}\right)$, and $1 Z_{\odot}\left(M_{\mathrm{w} 1}\right)$. The results are compared to homogeneous masses, and He-burning masses from Sect. 4.1.

We note that the mass-loss relation by Gräfener \& Hamann (2008) is obtained for stellar winds with an optical depth of the order of one, or higher, and that the $\mathrm{O}$ stars with lower luminosities in our sample may just lie outside the applicable range of this relation. Nevertheless we include them here, also to check the validity of the mass-loss relation.

In Fig. 6 we compare the resulting $M-L$ relation for $Z=$ $2 Z_{\odot}$, with the expected relation for homogeneous stars. We find a notable overall agreement between both $M-L$ relations. Morphologically, both relations show two branches in the $M-L$ diagram. The first branch, with higher luminosities for given wind mass, consists of H-deficient stars with subtypes WN7-9h

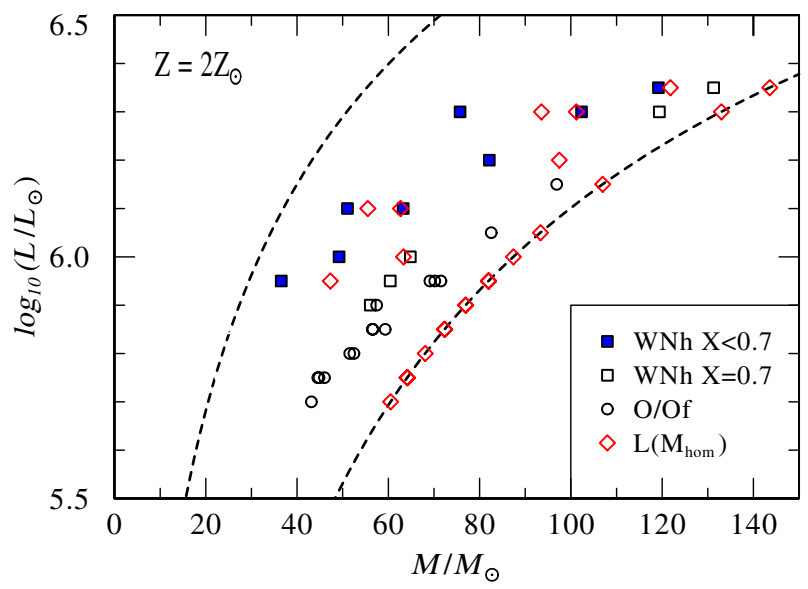

Fig. 6. Mass-luminosity relations $L(M)$ for the most massive stars in the Arches cluster, for an adopted metallicity of $Z=2 Z_{\odot}$. Plotted are observed luminosities, vs. different mass estimates. Red diamonds indicate $L\left(M_{\text {hom }}\right)$, with homogeneous masses $M_{\text {hom }}\left(L, X_{\mathrm{H}}\right)$ computed from Eqs. (11) and (12), with coefficients from row 11 in Table A.1. Black/blue symbols indicate $L\left(M_{\text {wind }}\right)$, with wind masses obtained from the mass-loss prescription by Gräfener \& Hamann (2008) (Eqs. (3-5)) for $Z=2 Z_{\odot}$. For the latter, we distinguish between WNh subtypes (squares), and O/Of subtypes (circles). Among these, blue filled symbols indicate $\mathrm{H}$-deficient (WNh) stars. The dashed lines indicate the HeMS (left), and the ZAMS (right) according to our relations in Sect. 4.1.

(blue filled symbols). The second branch, with lower luminosities, consists of stars with normal hydrogen surface composition (empty black symbols). This group covers spectral subtypes O4-6I, O4-6If+, and WN8-9h. Our theoretically predicted masses are indicated by red diamonds. Also they show two branches, one on the ZAMS (at lower luminosities), and one in the region of our previous high-luminosity branch.

For the theoretically predicted masses $M_{\mathrm{hom}}\left(L, X_{\mathrm{H}}^{\mathrm{s}}\right)$ (red diamonds) the interpretation is very simple. By definition, homogeneous stars with solar $\mathrm{H}$-abundance populate the ZAMS, while $\mathrm{H}$-deficient stars have a higher mean molecular weight, and thus display higher luminosities.

For the wind masses $M_{\mathrm{w} 2}$ (black/blue symbols), the difference between both branches mainly originates from the higher mass-loss rates of the WNh stars, and the temperature difference between the $\mathrm{WNh}$, and the $\mathrm{O}$ stars. For the H-deficient branch, which consists only of WNh subtypes, the derived wind masses are in very good overall agreement with the homogeneous masses. For the H-rich branch, the derived wind masses do not fit the homogeneous masses, but are systematically lower, as expected for stars that have just evolved away from the ZAMS. Again, we have to keep in mind that the stars with the lowest luminosities in this group, may lie beyond the applicable regime of the theoretical mass-loss relation.

For the H-deficient WNh stars, we only find a good quantitative agreement between empirically determined wind masses, and theoretically predicted homogeneous masses, if we assume a high metallicity of $2 Z_{\odot}$. A similar metallicity is indeed measured indirectly for the WNh stars in the Arches sample, based on their nitrogen abundances. However, more recent investigations of stars in the GC, favor a solar value (cf. Sect. 6.1). Using the mass-loss relation by Gräfener \& Hamann (2008) with solar Z, the derived masses are lower, but the morphological similarities remain (cf. Fig. 7). In this case the wind masses of both branches in the diagram imply an inhomogeneous stellar structure.

We conclude that, using the mass-loss prescription by Gräfener \& Hamann (2008), we find two branches in the $M-L$ 


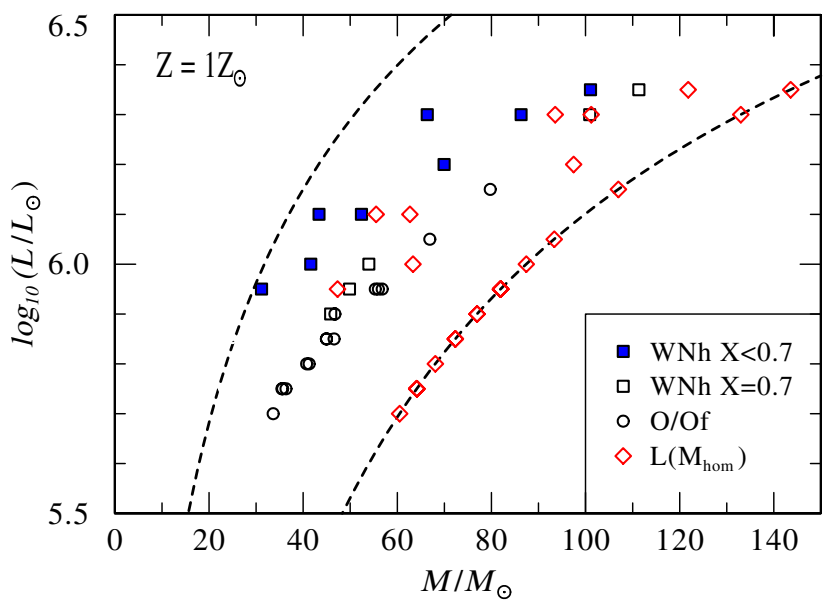

Fig. 7. Mass-luminosity relations $L(M)$ for the most massive stars in the Arches cluster, for an adopted metallicity of $Z=1 Z_{\odot}$. Symbols are the same as in Fig. 6.

diagram. The more luminous branch is populated by H-deficient WNh stars. Based on an adopted metallicity of $2 Z_{\odot}$, the derived wind masses for these stars would be in agreement with chemically homogeneous stars. Interestingly, these stars also show the strongest nitrogen enrichment (cf. Table 1), which points to a previous evolution with episodes of strong mixing, and/or strong mass loss, that could indeed lead to a quasi-homogeneous structure of these objects (cf. Sect. 6.4). The H-rich stars, on the other hand, populate a branch with lower luminosities, in agreement with stars that have undergone a normal evolution off the ZAMS.

At this point we want to note that our preference for a high $Z$ could mean that the mass-loss relation by Gräfener \& Hamann (2008) still underestimates the true mass loss rates. In this case the high $Z$ would compensate for lacking elements in the models. Another possibility would be, that the sample stars are not homogeneous, and thus $\Gamma_{\mathrm{e}}$ is underestimated by our approach. However, in any case the wind masses cover a plausible parameter range. Notably, almost all of the sample stars lie below the $\mathrm{He}$ main-sequence in the $M-L$ diagram. In agreement with our findings from Sect. 5.2, the largest part of the Arches cluster stars is thus most likely still in the phase of core hydrogen burning.

\section{Discussion}

In the present work we have investigated the mass-loss properties of the most massive stars in the Arches cluster. We have used a semi-empirical method that is based on observations, or more precisely, on the results of spectral analyses, and on theoretically predicted $M-L$ relations. As a result we found evidence for the existence of a largely $\Gamma$-dependent mass-loss relation, and obtained information about the evolutionary status of the Arches cluster stars.

In the following we discuss our results. We start with the question how the results are affected by potential uncertainties in the observed stellar parameters (Sect. 6.1). In Sect. 6.2 we discuss the implications for the mass loss properties of massive stars, and in Sect. 6.3 we reconsider the potential to obtain precise wind masses. Finally, in Sect. 6.4, we discuss the implications on the evolutionary status of the Arches cluster stars.

\subsection{Observational uncertainties}

Here we discuss to which extent our results are affected by uncertainties in the stellar parameters, that we use as input for our method. We give an overview of potential error sources, discuss

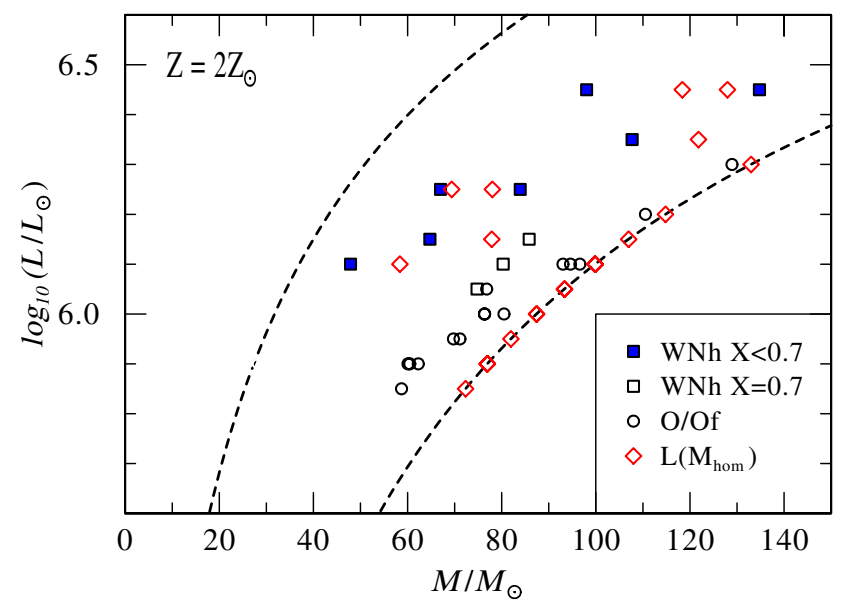

Fig. 8. Mass-luminosity relations $L(M)$ for the most massive stars in the Arches cluster, for an adopted metallicity of $Z=2 Z_{\odot}$. To investigate the influence of systematic uncertainties on our results, the observed luminosities have been artificially increased by $0.15 \mathrm{dex}$. To derive wind masses, the mass-loss rates have been increased according to $\dot{M} \propto L^{3 / 4}$, as expected for the results from recombination line analyses (cf., Hamann \& Koesterke 1998). The symbols are the same as in Fig. 6.

their quantitative importance, and their influence on the qualitative outcome of our work. Generally it can be said that, due to the steepness of the proposed $\dot{M}-\Gamma$ relation, our results are relatively insensitive to such uncertainties. In addition, most error sources are of systematic nature, and hardly affect the qualitative outcome of our work.

Martins et al. (2008) quote uncertainties of $\pm 3 \mathrm{kK}(2 \mathrm{kK})$ on $T_{\text {eff }}$ for O stars (WNLh), \pm 0.2 on $\log (L), 0.2(0.1)$ on $\log (\dot{M})$, and $\pm 50 \%( \pm 30 \%)$ on abundances. If these (rather conservative) error estimates would be of purely statistical nature, they would be devastating for our results. The fact that we actually find welldefined relations for the sample stars, indicates that potential errors are chiefly of systematic nature. The possibility that there are differences in the systematics between $\mathrm{O}$ stars, and WNh stars, could however influence our results, particularly regarding the temperature dependence in Sect. 5.2.

Also Crowther et al. (2010) recently pointed out that Martins et al. (2008) may have systematically under-estimated the stellar luminosities of the Arches cluster stars, by using a relatively small distance estimate $(m-M=14.4 \pm 0.1$, Eisenhauer et al. $2005)$, and a low foreground extinction $\left(A_{\mathrm{K}}=2.8 \pm 0.1\right.$ Stolte et al. 2002) for the GC. Using $m-M=14.5 \pm 0.1$ (Reid 1993), and $A_{\mathrm{K}}=3.1 \pm 0.1$ (Kim et al. 2006) Crowther et al. find slightly higher luminosities for the two brightest stars in the sample $\left(\log \left(L / L_{\odot}\right)=6.5\right.$ instead of 6.35$)$.

Although a systematic increase of $\log (L)$ by 0.15 dex would be significant, the $\Gamma_{\mathrm{e}}$, as derived from the mass-loss relations in Sect. 3, would hardly be affected. The reason is that spectroscopic mass-loss rates derived from recombination line analyses, as in Martins et al. (2008), scale with $\dot{M} \propto L^{0.75}$. As the luminosity scaling in our mass-loss relations is very similar (Eqs. (6), (7), and (3)), the derived $\Gamma_{\mathrm{e}}$ would nearly stay the same. This would however still result in uncertainties in the derived wind masses, basically with $M_{\text {wind }} \propto L\left(1+X_{\mathrm{H}}^{\mathrm{s}}\right)$ (cf. Eq. (8)). The effect of a systematic luminosity increase by 0.15 dex is illustrated in Fig. 8.

For the mass-loss relations in Sect. 5.2, a luminosity increase mainly results in a shift of the inferred mass-loss rates with $\dot{M} \propto$ $L^{0.75}$. In addition, the change of the slope of the $M-L$ relation changes the slope of the mass-loss relations, with respect to $\Gamma_{\mathrm{e}}$. 
E.g., based on relation No. 1 in Table A.1, an increases of 0.15 in $\log (L)$ for a star with $\log \left(L / L_{\odot}\right)=6.3$, and $X=0.7$ (i.e., $\left.\log \left(\Gamma_{\mathrm{e}}\right)=-0.386\right)$ causes an increase of $\Gamma_{\mathrm{e}}$ by 0.048 dex. For a star with $\log \left(L / L_{\odot}\right)=5.7\left(\log \left(\Gamma_{\mathrm{e}}\right)=-0.647\right)$, the same increase causes $\Gamma_{\mathrm{e}}$ to increase by 0.073 dex. Consequently, the exponents in our empirical mass-loss relations increase by a factor of $1+$ $(0.073-0.048) /(0.647-0.386)=1.1$.

Additional uncertainties in the mass-loss rates are due to wind clumping (with $\dot{M} \propto \sqrt{f_{\text {cl }}}$ ), and the unknown wind velocity field. Again, these would introduce a shift in $\dot{M}$, but only moderately affect the derived $\Gamma_{\mathrm{e}}$, because of the steepness of the proposed $\Gamma$-dependence. Based on the aforementioned uncertainties, we thus expect systematic effects on our quantitative results, particularly concerning mass-loss rates, and wind masses, but only moderate relative changes, i.e., our results stay qualitatively the same.

Apart from that, the metallicity $Z$ is of major importance. In contrast to the above, $Z$ does affect the $\Gamma$-dependence in our theoretical mass-loss relations, but not the observed properties of the sample stars. As discussed in Sect. 5.3, it thus affects the derived wind masses. In the relation by Gräfener \& Hamann (2008, Eq. (3)), the metallicity determines the value $\Gamma_{0}(Z)$ (Eq. (4)), which roughly marks the value of $\Gamma_{\mathrm{e}}$ for which the mass loss starts to increase due to the proximity to the Eddington limit. Moreover, the exponent $\beta(Z)$ defines the steepness of the massloss relation. Also Vink \& de Koter (2005) find a mass-loss dependence with $\dot{M} \propto Z^{0.68}$ for a typical Wolf-Rayet model. The reason for the strong $Z$-dependence lies in the dominant role of Fe line opacities in the radiative acceleration of hot star winds. The $Z$ dependence in the wind models thus chiefly reflects a dependence on the Fe abundance.

As mentioned before, the metallicity of the Arches cluster is still uncertain. It has been determined indirectly, on the basis of observed nitrogen surface abundances of WNh stars (Najarro et al. 2004; Martins et al. 2008). These stars are strongly nitrogen enriched, obviously by material which has been processed in the CNO cycle in the stellar core. This material is exposed at the stellar surface, presumably by mixing and/or mass loss. The observed $\mathrm{N}$ abundance thus provides a lower limit for the initial abundance of $\mathrm{C}+\mathrm{N}+\mathrm{O}$. For the WNh stars in the Arches cluster Najarro et al. (2004) find $X_{\mathrm{N}} \approx 1.6 \%$, and Martins et al. (2008) find an average of $X_{\mathrm{N}} \approx 1.7 \%$, with values reaching up to $X_{\mathrm{N}}=2.79 \%$. Both authors conclude that the initial metallicity of the Arches cluster is only slightly super-solar. However, taking into account the recent downward revision of the solar oxygen abundance (according to Asplund et al. 2005, 2009; Pereira et al. 2009 , it follows that $X_{\mathrm{C}+\mathrm{N}+\mathrm{O}, \odot} \approx 0.8 \%$ ), a metallicity of $2 Z_{\odot}$ seems more likely. As the solar Fe abundance has hardly been affected by this revision, a simple scaling would imply that $X_{\mathrm{Fe}} / X_{\mathrm{Fe}, \odot}=2$.

We note however that recent spectral analyses of LBVs (Najarro et al. 2009), and red supergiants (Davies et al. 2009), as well as earlier studies of cool stars in the GC region (Carr et al. 2000; Ramírez et al. 2000; Cunha et al. 2007) indicate a solar Fe abundance, and an increased abundance of alpha elements. The resulting uncertainty in the derived wind masses is of the order of $20 \%$ (see Table 1). Generally, radiatively driven winds become stronger for higher $Z$, i.e., the derived wind masses become systematically larger if a higher $Z$ is adopted. As the derived mass ratios are hardly affected, we do not expect a qualitative impact on our results from Sect. 5.3. The preference for a high Fe abundance in this work, particularly in the comparison in Fig. 5, may indicate that either the $\Gamma_{\mathrm{e}}$ in Sects. 5.2, and 5.3 are underestimated, due to the assumption of homogeneity, or the mass-loss predictions by Gräfener \& Hamann (2008) are still too low. In any case, we do not expect a qualitative impact on our results, as the differential stellar properties within the sample are hardly affected.

\subsection{The mass-loss properties of very massive stars}

The main result of the present study is the empirical confirmation of a $\Gamma$-dependent mass-loss relation for stars approaching the Eddington limit. This result is consistent with theoretical predictions (Gräfener \& Hamann 2006; Vink 2006; Gräfener \& Hamann 2008; Vink et al. 2011), as summarized in Sect. 3. A similar $\Gamma$-dependent relation has also been proposed for the winds of LBVs, by Vink \& de Koter (2002). When we express our empirical results in the form $\dot{M}\left(\Gamma_{\mathrm{e}}, T_{\star}, L, X_{\mathrm{H}}^{\mathrm{s}}\right)$, we find that the dependencies on $L$, and $X_{\mathrm{H}}^{\mathrm{s}}$ are very weak, indicating that $\Gamma_{\mathrm{e}}$, and potentially $T_{\star}$, are the physically most relevant parameters. The temperature dependence in Eq. (15) is in remarkable agreement with Gräfener \& Hamann (2008), however, based on the present data, this result seems not fully established.

The empirical confirmation of a $\Gamma$-dependence marks a paradigm shift in the way we think about the development of stellar mass loss through subsequent evolutionary phases. The physical effect that causes (LBV and) WR-type mass loss is the proximity to the Eddington limit. During their evolution, the cores of massive stars become chemically enriched, and their $L / M$ ratio increases. When Eddington factors of order unity are reached, a strong WR-type stellar wind will develop naturally. With the removal of the outer envelope, in combination with mixing processes, a hydrogen deficiency will develop at the stellar surface. It is therefore not the hydrogen deficiency that defines the onset of the WR phase, as is usually assumed in stellar evolution calculations. Rather, the WR-phase starts as soon as Eddington factors of order unity are reached.

This effect will have strong impact on the evolution of massive stars, in terms of conventional mass loss, but also in terms of loss of angular momentum (e.g., Langer 1998). For fast rotating stars, the Eddington parameter effectively increases near the equator, due to centrifugal forces. The effect of rotation on mass loss has been studied for OB stars, e.g. by Friend \& Abbott (1986); Pelupessy et al. (2000); Curé \& Rial (2004); Curé et al. (2005); Madura et al. (2007). Close to rotational breakup (i.e., close to the $\Omega$-limit), the latter two of these works find shallow wind solutions, for which the mass loss does not significantly exceed the mass loss without rotation (only by a factor of two, see Madura et al. 2007). For WR-type winds, the effect of rotation has not yet been investigated. The strong $\Gamma$-dependence however implies that the combined effect of rotation and high $\Gamma$ may still be efficient at high rotational speed (i.e., close to the $\Gamma \Omega$-limit). This effect may be of paramount importance for fast rotating WR stars, like the progenitors of long GRBs (Yoon \& Langer 2005). At the present stage this is however highly speculative.

In addition to that, the continuous transition between the $\mathrm{O} / \mathrm{Of}$, and WNh stages (cf. Fig. 5) has consequences for our theoretical understanding of the winds of very massive stars. Notably, this suggests that they have the same driving mechanism, namely radiation pressure. Nevertheless, there are important differences between O-star winds and WR-winds. While the former are expected to be optically thin at the sonic point, the latter are optically thick, i.e., the wind is initiated and already accelerated in part below the surface. The transition between these two regimes has been resolved by the model computations of Vink et al. (2011) with their different $\Gamma$-dependencies 
for the OB, and the WR regime (Eqs. (6) and (7)). Vink et al. find that this transition occurs for models with a wind efficiency $\eta=\dot{M} v_{\infty} /(L / c) \approx 1$, which roughly corresponds to the point where the winds become optically thick.

The properties of optically thick, WR-type winds have been suggested to be determined by the so-called Fe-opacity peaks in deep atmospheric layers, close to the sonic point (e.g., Pistinner \& Eichler 1995; Heger \& Langer 1996; Schaerer 1996; Nugis \& Lamers 2002). This has been confirmed by WR wind models of Gräfener \& Hamann $(2005,2008)$, who proposed that the formation of optically thick winds is supported close to the Eddington limit, because the density scale height in the deep atmospheric layers increases. In contrast to this, OB star winds are thought to be dominated by the outer wind physics. Within the classical theory of radiatively driven winds (Castor et al. 1975), their wind properties are determined at a critical point with a much higher wind speed, which is typically of the order of the escape speed. The wind energy at this point is thus of the same order of magnitude as the gravitational energy. Small changes in the (effective) gravitational potential thus only have small influence on the mass loss of OB stars, i.e., the $\Gamma$-dependence is expected to be weak. The fact that we find observational evidence for a strong $\Gamma$-dependence of WR mass loss, in agreement with the model predictions, shows that there is indeed a substantial difference in the way the mass-loss rates of OB and WR stars are determined.

A strong temperature dependence of WR-type mass loss would support the importance of the deep layers for the wind physics of WR stars. Gräfener \& Hamann (2008) predict a similar dependence, with $\dot{M} \propto T_{\star}^{-3.5}$. They explain this dependence by the necessity that the critical point of the equation of motion needs to be located in a specific temperature regime, and thus at higher optical depths for cooler stars. They also show that this temperature dependence is in agreement with the observed line strengths of galactic WNL stars (cf. Fig. 2 in Gräfener \& Hamann 2008). Quantitatively, the temperature dependence might be of major importance because it leads into phases of extremely high mass loss, potentially even to a smooth transition into the LBV phase. We note, however, that our empirical result arises from a comparison of the hotter O/Of stars vs. the cooler WNh stars. Particularly for the low-luminosity end of the O star sample we concluded in Sect. 5.3 that they might have a systematically different internal structure than the WNh stars. Moreover, for the relatively weak winds of these objects, the concept of $\Gamma$-dependent mass loss might be questionable. The fact that we actually find a relation that includes these objects is thus rather surprising. Because of these concerns it would be desirable to back up this result by complementary studies of WNh stars in different temperature regimes.

In Fig. 5 we compare our empirical relation (Eq. (15)) with theoretical mass-loss predictions by Gräfener \& Hamann (2008) for different metallicities $Z$. For a high $Z$ of $2 Z_{\odot}$, we find a good quantitative agreement between theory and observation. Qualitatively, the slope of the empirical $\Gamma$-dependence is very well reproduced by the models. The same holds for the relation by Vink et al. (2011). Their exponent of 3.99 in Eq. (7) matches the exponent in Eq. (15) very precisely. The major difference between the model predictions in Sect. 3 thus lies in the strong temperature dependence that is predicted by Gräfener \& Hamann (2008).

Summing up our results, we expect that massive stars close to the Eddington limit tend to form WR-type winds with distinct properties from classical radiatively driven winds. In the WR regime we find mass-loss relations of the form $\dot{M}\left(\Gamma_{\mathrm{e}}, T_{\star}, L, X_{\mathrm{H}}^{\mathrm{s}}\right)$ which fundamentally depend on the Eddington parameter $\Gamma_{\mathrm{e}}$. Our theoretical models predict 1) a strong $\Gamma$-dependence, 2) a weak dependence on $L$, and $X_{\mathrm{H}}^{\mathrm{s}}$, and 3) a strong temperature dependence, according to Gräfener \& Hamann (2008). Empirically, point 1), and 2) are convincingly confirmed in our present study. The important temperature dependence 3), is in agreement with our present results, however, demands for further empirical confirmation.

\subsection{Wind masses for very massive stars}

In Sect. 5.3 we used the mass-loss relations by Gräfener \& Hamann (2008) to derive the present masses of the Arches cluster stars, based on their observed mass-loss rates. The potential to estimate stellar masses in this way is a very interesting aspect of this work, in particular because our mass estimates are fundamentally different from wind masses that have previously been obtained for OB stars (e.g., Kudritzki et al. 1992).

In Sect. 6.2, we already discussed that the dynamics of classical radiation-driven winds is dominated by the outer part of the wind, while WR-type winds are strongly influenced by deep atmospheric layers. Classical OB stars thus show a well-defined relation between their escape velocity at the stellar surface, and their terminal wind velocity, that helps to determine their masses (Kudritzki \& Puls 2000; Prinja \& Massa 1998; Lamers et al. 1995). Only Vink \& de Koter (2002) previously used a $\Gamma$-dependent mass-loss relation to constrain the stellar mass of the LBV AG Car, however, their models still depend on the outer wind physics, and thus on the ratio $v_{\infty} / v_{\text {esc }}$.

WR-type winds depend mostly on the inner wind physics. We have seen that this leads to a steep $\Gamma$-dependence for stars that are close to the Eddington limit, i.e., we are capable to determine precise $\Gamma_{\mathrm{e}}$, without consideration of the terminal wind velocity. This method is very promising, because the masses of stars close to the Eddington limit are already well known, simply because $\Gamma_{\mathrm{e}}$ is of the order of one. The wind models thus only need to provide an "improved guess" of $\Gamma_{\mathrm{e}}$. Moreover, observational uncertainties are of minor importance because of the steepness of the $\Gamma$-dependence. The main uncertainties arise from the models, chiefly from the contribution of metal lines to the "physical" Eddington factor $\Gamma(r)$. However, even if this contribution would be systematically wrong, the models still provide reliable mass ratios, i.e., the mass-loss relation can be calibrated with observations. In the present work we have performed a first step towards such a calibration. It seems, however, that the remaining uncertainties in the metallicity, and the distance/extinction towards the Arches cluster demand for complementary studies in different environments.

\subsection{Evolutionary status of the Arches cluster stars}

As a by-product of our present study we obtain information about the internal structure, and thus about the evolutionary status of the WNh stars in the Arches cluster. Most importantly, we find direct evidence that the largest part of these objects is still in the phase of core hydrogen burning. In Fig. 4 we have shown that, if the stars were in the phase of core He-burning, 9 of the $13 \mathrm{WNh}$ stars in the sample would have Eddington factors $\Gamma_{\mathrm{e}}>0.75$ (cf. also Table 1). Taking into account that $\Gamma(r)>\Gamma_{\mathrm{e}}$ (Eq. (2)), this would imply that these stars are extremely close, or even above the physical Eddington limit. The reason for this are the high hydrogen surface abundances of these stars (up to solar). 
The $M-L$ relations used in this work are based on the assumption of a simple, chemically homogeneous internal structure of the sample stars. In Sects. 5.2 and 5.3 we find indications that this assumption may indeed be fulfilled by most of the WNh stars in our sample. However, due to uncertainties in the observational data, and the models, we cannot draw firm conclusions at this stage.

E.g., we found in Sect. 5.3 that the wind masses of the $\mathrm{H}$-deficient WNh stars are in good overall agreement with the expected masses of chemically-homogeneous stars (cf. Fig. 6). This result however depends on the adopted metallicity in our mass-loss relation. In Sect. 5.2, we found evidence for chemical homogeneity, based on mass ratios, which are much more certain (cf. Sects. 6.1 and 6.3). Figures 3 and 4 show that, under the assumption of chemical homogeneity, the relative masses of the sample stars adjust in a way that we observe a smooth $\dot{M}\left(\Gamma_{\mathrm{e}}\right)$ relation. Under the assumption of core He-burning, i.e., the completely in-homogeneous case, we obtain differences in $\dot{M}$ by a factor of $\sim 10$ for similar $\Gamma_{\mathrm{e}}$, particularly for $\mathrm{H}$-deficient $\mathrm{WNh}$ stars. This suggests that most of the H-deficient WNh stars in the Arches cluster are close to homogeneity, although a similar behaviour may also originate from a stellar sample where all stars have a similar degree of inhomogeneity.

A chemically homogeneous structure is expected for single, fast rotating massive stars, e.g., according to evolutionary models by, Maeder (1987); Yoon et al. (2006); Brott et al. (2011). Note, however, that fast rotation is no mandatory condition to achieve a nearly homogeneous stellar structure. Because the convective cores of very massive stars are very large, their remaining radiative envelopes can easily be stripped off by strong mass loss (cf., Yungelson et al. 2008). E.g., for the WNh stars in our sample we find mass-loss timescales of $\tau_{\text {wind }}=M / \dot{M} \approx 1 . .6 \times 10^{6} \mathrm{yr}$, similar to their evolutionary timescales of $\tau_{\text {nuc }}=7.2 \times 10^{10} \mathrm{yr}$. $\left(M / M_{\odot}\right) /\left(L / L_{\odot}\right) \approx 3 . .5 \times 10^{6} \mathrm{yr}$. In addition, other scenarios, like mergers due to frequent stellar collisions in dense clusters (e.g., Portegies Zwart et al. 1999) might be considered in this context.

An argument against chemical homogeneity are the low surface temperatures of the Arches cluster stars $(30-35 \mathrm{kK})$. Because of their high mean molecular weight, homogeneous stars are expected to be very compact, and to show very high surface temperatures. Martins et al. (2009) indeed found evidence for homogeneous evolution for an extremely early WN3h star in the SMC, based on its high temperature $\left(T_{\star}=65 \mathrm{kK}\right)$. This star is clearly located to the left of the main sequence, but has still $50 \%$ hydrogen at its surface, in agreement with evolutionary tracks for fast rotating quasi homogeneous stars by Meynet $\&$ Maeder (2005). So why are the Arches cluster stars so much cooler?

In principle, their temperatures may indicate that the Arches stars are evolved main sequence stars, and not homogeneous. However, Ishii et al. (1999) found that very massive homogeneous stars at high metallicities can have inflated stellar envelopes. This effect is expected to occur for stars close to the Eddington limit, and to be enhanced by fast rotation (Maeder et al. 2008). The inflation is caused by the Fe opacity peak at temperatures around $160 \mathrm{kK}$. The effect is thus metallicity dependent. Note that the same opacity peak is expected to drive the inner winds of early WR subtypes (Nugis \& Lamers 2002; Gräfener \& Hamann 2005). However, for late WR subtypes, like the Arches stars, the opacity peak is located below the wind driving zone, and may thus affect the stellar envelope. The existence of the envelope extension has been questioned by Petrovic et al. (2006) because in their hydrodynamic stellar structure models the effect was suppressed by mass loss. It is thus not clear whether the envelope inflation occurs in nature, but it is in line with the observed low temperatures of many H-free WR stars (e.g., Hamann et al. 2006), and the tendency that late WR subtypes are preferentially found in high metallicity environments (e.g., Crowther 2007).

\section{Conclusions}

Based on a semi-empirical study of the most massive stars in the Arches cluster we could confirm key properties of WR-type stellar winds that have been predicted in our previous theoretical studies (Gräfener \& Hamann 2008; Vink et al. 2011). We find that mass loss is enhanced close to the Eddington limit, with a dependence of the form $\dot{M}\left(\Gamma_{\mathrm{e}}, T_{\star}, L, X_{\mathrm{H}}^{\mathrm{s}}, Z\right)$ that shows 1$)$ a strong dependence on the Eddington factor $\Gamma_{\mathrm{e}}, 2$ ) a weak dependence on the luminosity $L$, and the hydrogen surface abundance $X_{\mathrm{H}}^{\mathrm{s}}$, and 3) a strong increase of mass loss for decreasing stellar temperatures $T_{\star}$. Due to the properties of the sample, the latter is however not fully established. The qualitative agreement between models and observations suggests that the mass-loss properties of WR stars are determined in analogy with the models, by the influence of the $\mathrm{Fe}$-opacity peaks in deep atmospheric layers.

The strong $\Gamma$-dependence marks an important paradigm shift with respect to previous, luminosity-dependent relations, that are based on purely empirical studies (Nugis \& Lamers 2000; Hamann et al. 2006). The latter are widely used, e.g., in stellar evolution models, where the WR-phase is usually identified by a hydrogen-deficient surface composition. According to our work, the physically relevant parameter, which causes WR-type mass loss, is the proximity to the Eddington limit. A hydrogen deficiency then occurs naturally as a consequence of the strong mass loss, potentially in combination with rotational mixing.

As a by-product of our work we obtain information on the evolutionary status of the sample objects. We find strong evidence that the luminous WNh stars in the Arches cluster are not evolved objects, but mostly very massive, hydrogen-burning stars, supporting the picture that the most massive stars are WNh stars in young stellar clusters (e.g., de Koter et al. 1997; Crowther et al. 2010).

The mass loss in the WR phase is of paramount importance for the evolution, and the death of massive stars, as well as the formation of long GRBs. With our present work we have made a first step towards a more physically motivated description of WR-type mass loss, incorporating the dependence on the Eddington factor $\Gamma_{\mathrm{e}}$. Other predicted dependencies, e.g. on the stellar temperature, and the metallicity, demand for similar studies of large stellar samples in different environments. Combined observational and theoretical programs, like the ongoing VLTFLAMES Tarantula Survey (Evans et al. 2011; Bestenlehner et al. 2011; Bestenlehner et al., in prep.), will give improved insight into exactly these questions in the near future.

\section{Appendix A: Mass-luminosity relations for a broad range of stellar masses}

In Sect. 4 of this work we provided fitting formulae for mass-luminosity relations $L\left(M, X_{\mathrm{H}}\right)$, and the corresponding masses $M_{\mathrm{hom}}\left(L, X_{\mathrm{H}}\right)$, and $M_{\mathrm{Heb}}(L)$ for massive, chemicallyhomogeneous stars. We focused on a mass range of 12-250 $M_{\odot}$, which covers the observed properties of the most massive stars known. Within this mass range we obtained a very good fit quality for $L\left(M, X_{\mathrm{H}}\right)$, with a fitting error of up to $0.02 \mathrm{dex}$. The resulting fitting relations are however strictly limited to this mass 
Table A.1. Mass-luminosity relations for chemically-homogeneous stars.

\begin{tabular}{|c|c|c|c|c|c|c|c|c|c|c|c|c|}
\hline No. & Ev. ph. & Eq. & Mass range & $F_{1}$ & $F_{2}$ & $F_{3}$ & $F_{4}$ & $F_{5}$ & $F_{6}$ & $F_{7}$ & $F_{8}$ & $F_{9}$ \\
\hline 1 & H-b. & 9 & $12-250 M_{\odot}$ & 2.875 & -3.966 & 2.496 & 2.652 & -0.310 & -0.511 & & & \\
\hline 2 & H-b. & 9 & $2-100 M_{\odot}$ & 1.967 & -2.943 & 3.755 & 1.206 & -0.727 & -0.026 & & & \\
\hline 3 & H-b. & 9 & $60-4000 M_{\odot}$ & 3.862 & -2.486 & 1.527 & 1.247 & -0.076 & -0.183 & & & \\
\hline 4 & H-b. & B.7 & 2-30 $M_{\odot}$ & -2.688 & -7.843 & 2.471 & 2.758 & -0.233 & -0.747 & & & \\
\hline 5 & H-b. & B.7 & $12-4000 M_{\odot}$ & -2.416 & -5.118 & 1.869 & -0.400 & 0.064 & 0.050 & & & \\
\hline 6 & He-b. & 10 & $8-250 M_{\odot}$ & 3.017 & 2.446 & -0.306 & & & & & & \\
\hline 7 & He-b. & 10 & $0.6-100 M_{\odot}$ & 2.635 & 2.986 & -0.488 & & & & & & \\
\hline 8 & He-b. & 10 & $60-1000 M_{\odot}$ & 3.826 & 1.619 & -0.099 & & & & & & \\
\hline 9 & He-b. & B. 8 & $0.3-100 M_{\odot}$ & -2.204 & 1.831 & 0.149 & & & & & & \\
\hline 10 & He-b. & B. 8 & $12-500 M_{\odot}$ & -1.676 & 1.075 & 0.404 & & & & & & \\
\hline 11 & H-b. & 11,12 & $12-250 M_{\odot}$ & 4.026 & 4.277 & -1.0 & 25.48 & 36.93 & -2.792 & -3.226 & -5.317 & 1.648 \\
\hline 12 & H-b. & 11,12 & $2-100 M_{\odot}$ & 2.582 & 0.829 & -1.0 & 9.375 & 0.333 & 0.543 & -1.376 & -0.049 & 0.036 \\
\hline 13 & H-b. & 11,12 & $60-4000 M_{\odot}$ & 10.05 & 8.204 & -1.0 & 151.7 & 254.5 & -11.46 & -13.16 & -31.68 & 2.408 \\
\hline 14 & H-b. & B.13, B.14 & $2-30 M_{\odot}$ & 5.303 & 5.918 & -1.0 & 16.58 & -4.292 & -72.89 & -7.881 & -13.76 & 3.206 \\
\hline 15 & H-b. & B.13, В.14 & $12-4000 M_{\odot}$ & -14.60 & 3.125 & 1.0 & 251.0 & 15.63 & 72.24 & 18.20 & 12.21 & 0.781 \\
\hline 16 & He-b. & 13 & $8-250 M_{\odot}$ & 3.997 & -1.0 & 25.83 & -3.268 & & & & & \\
\hline 17 & $\mathrm{He}-\mathrm{b}$. & 13 & $0.6-100 M_{\odot}$ & 3.059 & -1.0 & 14.76 & -2.049 & & & & & \\
\hline 18 & He-b. & 13 & $60-1000 M_{\odot}$ & 8.177 & -1.0 & 105.5 & -10.10 & & & & & \\
\hline 19 & He-b. & B. 15 & $0.3-100 M_{\odot}$ & -6.144 & 1.0 & 52.54 & 6.711 & & & & & \\
\hline 20 & $\mathrm{He}-\mathrm{b}$. & B. 15 & $12-500 M_{\odot}$ & -1.330 & 1.0 & 5.919 & 2.475 & & & & & \\
\hline
\end{tabular}

Notes. The table describes the mass-luminosity relations $L\left(M, X_{\mathrm{H}}\right)$, and $M\left(L, X_{\mathrm{H}}\right)$ that are derived in this work, as well as the relations $\Gamma_{\mathrm{e}}\left(M, X_{\mathrm{H}}\right)$, and $M\left(\Gamma_{\mathrm{e}}, X_{\mathrm{H}}\right)$ in the appendix. For each relation we give the assumed evolutionary phase, the corresponding equation numbers, the relevant mass range, and the numeric coefficients $F_{n}$.

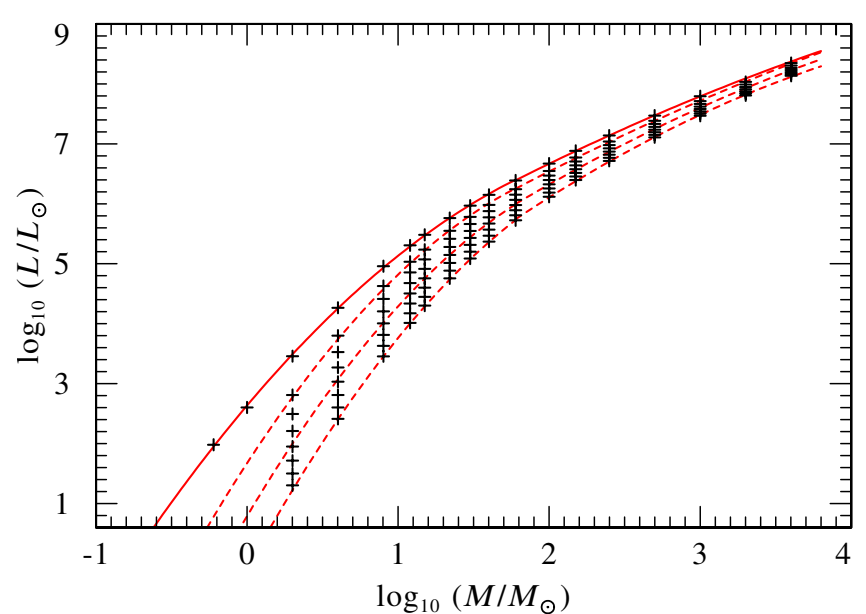

Fig. A.1. Homogeneous stellar structure models for the mass range 0.6$4000 M_{\odot}$ (black symbols). The models are computed for hydrogen mass fractions $X_{\mathrm{H}}=0.0-0.7$, in steps of 0.1 (from top to bottom). Dashed red lines indicate the $M-L$ relations for $X_{\mathrm{H}}=0.1,0.4$, and 0.7 according to Eq. (9) and Table A.1, and the solid red line corresponds to pure $\mathrm{He}$ models (Eq. (10) and Table A.1).

range. The reason is that just within this range the $M-L$ relation changes from a power law $L \propto M^{\eta}$, with $\eta \approx 3.8$ for low masses, to a nearly linear relation with $\eta \rightarrow 1$ for very massive stars. Our fits in Sect. 4 describe this "bending" of the $M-L$ relation very well, but are not capable of reproducing the asymptotic behaviour for higher and lower masses. We therefore decided to provide separate fitting relations in this appendix, that cover the range of 2-4000 $M_{\odot}$ for H-burning stars, and 0.6-1000 $M_{\odot}$ for He-burning stars.

In analogy to Sect. 4 , we obtain separate fits to our computational results for H-burning stars with $X_{\mathrm{H}}=0.1-0.7$, and $\mathrm{He}-$ burning stars $\left(X_{\mathrm{H}}=0\right)$. The resulting relations have the same

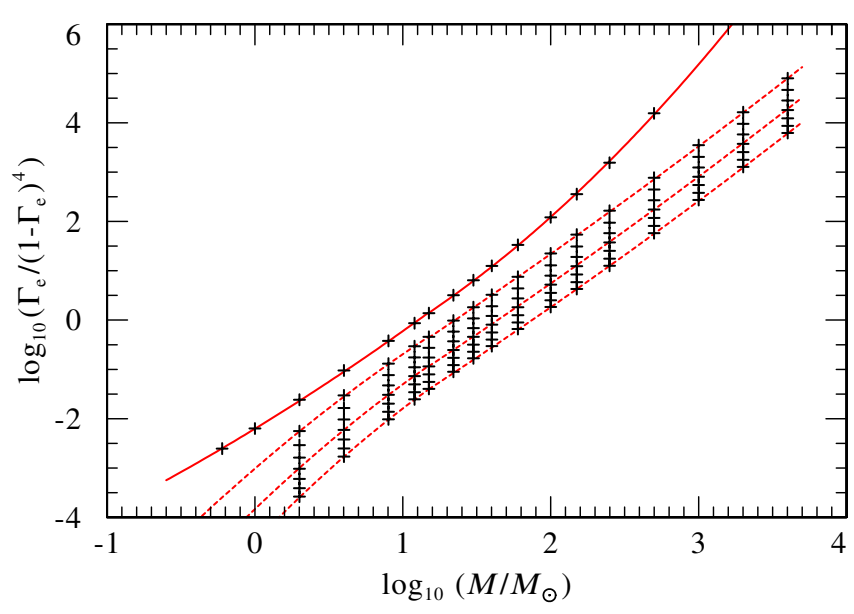

Fig. A.2. Eddington parameters for the same models as in Fig. A.1. The quantity $G_{4}=\Gamma_{\mathrm{e}} /\left(1-\Gamma_{\mathrm{e}}\right)^{4}$ is theoretically expected to follow a relation with $G_{4} \propto M^{2}$ (see Eq. (B.5)). The symbols in this plot are the same as in Fig. A.1.

form as Eqs. (9) and (10) in Sect. 4. Moreover, we obtain separate relations for the steep low-mass part, and the flat high-mass part of the $M-L$ relation. The corresponding coefficients are given in Table A.1. The maximum fitting errors in $\log \left(L / L_{\odot}\right)$ are 0.05 for H-burning stars with masses of $2-100 M_{\odot}, 0.02$ for $\mathrm{H}-$ burning stars with $60-4000 M_{\odot}, 0.03$ for He-burning stars with 0.6-100 $M_{\odot}$, and 0.003 for He-burning stars with 60-1000 $M_{\odot}$. The results are displayed in Fig. A.1.

Inverting these relations, we obtain $M_{\mathrm{hom}}\left(L, X_{\mathrm{H}}\right)$, and $M_{\mathrm{Heb}}(L)$ in the same way as in $\operatorname{Sect}$. 4, i.e., $M_{\mathrm{hom}}\left(L, X_{\mathrm{H}}\right)$ is given by Eqs. (11) and (12), and $M_{\mathrm{Heb}}(L)$ by Eq. (13), with coefficients from Table A.1. 


\section{Appendix B: Asymptotic behaviour of the Eddington factor for large stellar masses}

For the highest stellar masses, the $M-L$ relation has to become nearly linear, because $\Gamma_{\mathrm{e}} \rightarrow 1$. As $\Gamma_{\mathrm{e}}$ itself hardly changes in this range, the value of $1-\Gamma_{\mathrm{e}}$ becomes physically more relevant. Our previous fitting relations, however, chiefly focus on $M$, and $L$, i.e., on the correct reproduction of $\Gamma_{\mathrm{e}}$. The resulting value of $1-\Gamma_{\mathrm{e}}$ can be significantly affected by small uncertainties due to fitting errors. E.g., for the largest masses of up to $4000 M_{\odot}$, our relation in Eq. (9) reproduces the stellar luminosity and thus $\Gamma_{\mathrm{e}}$ rather precisely, but the error in $1-\Gamma_{\mathrm{e}}$ amounts to $0.1 \mathrm{dex}$. To improve on this we take advantage of a theoretically predicted scaling relation that has recently been pointed out by Owocki \& Shaviv (2011), and goes back to the original work by Eddington (1918).

With Eddington's original definition

$(1-\beta) \equiv \frac{P_{\mathrm{rad}}}{P}=\frac{a T^{4}}{3 P}$

the total pressure $P$ due to gas + radiation can be written in the form

$P=P_{\text {gas }}+P_{\text {rad }}=\frac{\mathcal{R}}{\mu} \rho T+\frac{a}{3} T^{4}=\left(\frac{3 \mathcal{R}^{4}}{a \mu^{4}}\right)^{\frac{1}{3}}\left(\frac{(1-\beta)}{\beta^{4}}\right)^{\frac{1}{3}} \rho^{\frac{4}{3}}$.

Assuming $\beta$ is a constant, this relation leads to a polytropic equation of state with $P \propto \rho^{4 / 3}$, which is the basis of the Eddington Standard Model.

Eddington's assumption that $\beta$ is a constant, holds very well in large parts of the stellar envelope where the energy transport is dominated by radiation, and where the opacity is dominated by the constant electron scattering opacity $\chi_{\mathrm{e}}$. In that case it follows from the equation of radiative diffusion that

$\frac{\partial P_{\mathrm{rad}}}{\partial r}=\frac{\chi_{\mathrm{e}} \rho L}{4 \pi r^{2} c}=\Gamma_{\mathrm{e}} \frac{G M(r) \rho}{r^{2}}=\Gamma_{\mathrm{e}} \frac{\partial P}{\partial r}$.

The latter equality follows from the definition of the classical Eddington factor $\Gamma_{\mathrm{e}}$ (Eq. (1)), and the equation of hydrostatic equilibrium. Under the assumption that $\Gamma_{\mathrm{e}}$ is a constant, Eq. (B.3) is just the derivative of the relation $P_{\mathrm{rad}}=\Gamma_{\mathrm{e}} P$, i.e., it becomes equivalent to Eq. (B.1). We thus have $P_{\text {rad }} / P=(1-\beta)=$ $\Gamma_{\mathrm{e}}$, and with Eq. (B.2),

$P^{3}=\left(\frac{3 \mathcal{R}^{4}}{a \mu^{4}}\right)\left(\frac{\Gamma_{\mathrm{e}}}{\left(1-\Gamma_{\mathrm{e}}\right)^{4}}\right) \rho^{4}$.

Here we are interested in how $\Gamma_{\mathrm{e}}$ changes qualitatively with the stellar mass $M$. For this purpose we express $P$, and $\rho$ in Eq. (B.4) by two-point scaling relations in $M$, and $R$. From hydrostatic equilibrium it follows directly that $P / R \approx G M \rho / R^{2}$. With $\rho \approx$ $M / R^{3}$ this leads to $P \approx M^{2} / R^{4}$. Using the last two relations with Eq. (B.4), we obtain the approximate scaling relation

$\frac{\Gamma_{\mathrm{e}}}{\left(1-\Gamma_{\mathrm{e}}\right)^{4}} \propto \frac{P^{3}}{\rho^{4}} \propto M^{2}$

Based on this simple scaling law we obtain very precise fitting relations for the quantity

$G_{4} \equiv \frac{\Gamma_{\mathrm{e}}}{\left(1-\Gamma_{\mathrm{e}}\right)^{4}}$
The resulting fits to our computational results are plotted in Fig. A.2. For H-burning, chemically-homogeneous stars with $X_{\mathrm{H}}=0.1-0.7$ we use a relation of the form

$$
\begin{aligned}
\log \left(G_{4}\right)= & {\left[F_{1}+F_{2} \log \left(1+X_{\mathrm{H}}\right)\right] } \\
& +\left[F_{3}+F_{4} \log \left(1+X_{\mathrm{H}}\right)\right] \log \left(M / M_{\odot}\right) \\
& +\left[F_{5}+F_{6} \log \left(1+X_{\mathrm{H}}\right)\right] \log \left(M / M_{\odot}\right)^{2} .
\end{aligned}
$$

In Table A. 1 we give the coefficients $F_{1}-F_{6}$, for the mass ranges 2-30 $M_{\odot}$, and 12-4000 $M_{\odot}$. For pure He stars $\left(X_{\mathrm{H}}=0\right)$ we use

$\log \left(G_{4}\right)=F_{1}+F_{2} \log (M)+F_{3} \log (M)^{2}$,

with coefficients $F_{1}-F_{3}$ from Table A.1, for the mass ranges of $0.3-100 M_{\odot}$, and $12-500 M_{\odot}$. The maximum fitting error in $\log \left(G_{4}\right)$ for relations (B.7 and B.8) is 0.03 .

For given $G_{4}, \Gamma_{\mathrm{e}}$ can be obtained from the solution of Eq. (B.6)

$\Gamma_{\mathrm{e}}=1-\frac{C}{D^{1 / 6}} \sqrt{\frac{E}{G_{4}}}\left(\sqrt{\frac{3 \sqrt{D G_{4}}}{C E^{3 / 2}}-1}-1\right)$,

with the definitions

$C=\sqrt{1 / 12}$

$D=\left(C / 3 \sqrt{256 G_{4}+27}+1 / 2\right) / G_{4}^{2}$,

$E=3 G_{4} D^{2 / 3}-4$

Finally, we obtain relations for the corresponding masses $M_{\text {hom }}\left(G_{4}, X_{\mathrm{H}}\right)$, and $M_{\mathrm{Heb}}\left(G_{4}\right)$ from Eqs. (B.7) and (B.8).

$\log \left(M_{\mathrm{hom}} / M_{\odot}\right)=\frac{F_{1}+F_{2} \log \left(1+X_{\mathrm{H}}\right)+F_{3} \sqrt{g}}{1+F_{9} \log \left(1+X_{\mathrm{H}}\right)}$,

with

$g=F_{4}+F_{5} \log \left(G_{4}\right)+F_{6} \log \left(1+X_{\mathrm{H}}\right)^{2}$

$$
+\left(F_{7}+F_{8} \log \left(G_{4}\right)\right) \log \left(1+X_{\mathrm{H}}\right)
$$

and

$\log \left(M_{\mathrm{Heb}} / M_{\odot}\right)=F_{1}+F_{2} \sqrt{F_{3}+F_{4} \log \left(G_{4}\right)}$.

The coefficients $F_{n}$ are given in Table A.1.

\section{References}

Asplund, M., Grevesse, N., \& Sauval, A. J. 2005, in Cosmic Abundances as Records of Stellar Evolution and Nucleosynthesis, ed. T. G. Barnes III, \& F. N. Bash (San Francisco: ASP), ASP Conf. Ser., 336, 25 Asplund, M., Grevesse, N., Sauval, A. J., \& Scott, P. 2009, ARA\&A, 47, 481 Bestenlehner, J. M., Vink, J. S., Gräfener, G., et al. 2011, A\&A, 530, L14 Bonanos, A. Z., Stanek, K. Z., Udalski, A., et al. 2004, ApJ, 611, L33 Brott, I., de Mink, S. E., Cantiello, M., et al. 2011, A\&A, 530, A115

Carr, J. S., Sellgren, K., \& Balachandran, S. C. 2000, ApJ, 530, 307 Castor, J. I., Abbott, D. C., \& Klein, R. I. 1975, ApJ, 195, 157 Chiappini, C., Hirschi, R., Meynet, G., et al. 2006, A\&A, 449, L27 Crowther, P. A. 2007, ARA\&A, 45, 177

Crowther, P. A., \& Dessart, L. 1998, MNRAS, 296, 622

Crowther, P. A., \& Smith, L. J. 1997, A\&A, 320, 500

Crowther, P. A., Smith, L. J., Hillier, D. J., \& Schmutz, W. 1995, A\&A, 293, 427

Crowther, P. A., Schnurr, O., Hirschi, R., et al. 2010, MNRAS, 408, 731

Cunha, K., Sellgren, K., Smith, V. V., et al. 2007, ApJ, 669, 1011

Curé, M., \& Rial, D. F. 2004, A\&A, 428, 545

Curé, M., Rial, D. F., \& Cidale, L. 2005, A\&A, 437, 929

Davies, B., Origlia, L., Kudritzki, R., et al. 2009, ApJ, 694, 46

de Koter, A., Heap, S. R., \& Hubeny, I. 1997, ApJ, 477, 792 
Eddington, A. S. 1918, ApJ, 48, 205

Eisenhauer, F., Genzel, R., Alexander, T., et al. 2005, ApJ, 628, 246

Evans, C. J., Taylor, W. D., Hénault-Brunet, V., et al. 2011, A\&A, 530, A108

Friend, D. B., \& Abbott, D. C. 1986, ApJ, 311, 701

Gräfener, G., \& Hamann, W.-R. 2005, A\&A, 432, 633

Gräfener, G., \& Hamann, W.-R. 2006, in Stellar Evolution at Low Metallicity:

Mass Loss, Explosions, Cosmology, ed. H. J. G. L. M. Lamers, N. Langer, T.

Nugis, \& K. Annuk (San Francisco: ASP), ASP Conf. Ser., 353, 171

Gräfener, G., \& Hamann, W.-R. 2008, A\&A, 482, 945

Gräfener, G., Koesterke, L., \& Hamann, W.-R. 2002, A\&A, 387, 244

Hamann, W.-R., \& Koesterke, L. 1998, A\&A, 335, 1003

Hamann, W.-R., Gräfener, G., \& Liermann, A. 2006, A\&A, 457, 1015

Hansen, C. J., \& Kawaler, S. D. 1994, Stellar Interiors, Physical Principles, Structure, and Evolution (Secaucus, New Jersey, USA: Springer)

Heger, A., \& Langer, N. 1996, A\&A, 315, 421

Heger, A., Fryer, C. L., Woosley, S. E., Langer, N., \& Hartmann, D. H. 2003 , ApJ, 591, 288

Iglesias, C. A., \& Rogers, F. J. 1996, ApJ, 464, 943

Ishii, M., Ueno, M., \& Kato, M. 1999, PASJ, 51, 417

Kim, S. S., Figer, D. F., Kudritzki, R. P., \& Najarro, F. 2006, ApJ, 653, L113

Kudritzki, R., \& Puls, J. 2000, ARA\&A, 38, 613

Kudritzki, R., Hummer, D. G., Pauldrach, A. W. A., et al. 1992, A\&A, 257, 655

Lamers, H. J. G. L. M., Snow, T. P., \& Lindholm, D. M. 1995, ApJ, 455, 269

Langer, N. 1989, A\&A, 210, 93

Langer, N. 1998, A\&A, 329, 551

Lauterborn, D., Refsdal, S., \& Weigert, A. 1971, A\&A, 10, 97

Madura, T. I., Owocki, S. P., \& Feldmeier, A. 2007, ApJ, 660, 687

Maeder, A. 1987, A\&A, 178, 159

Maeder, A., Georgy, C., \& Meynet, G. 2008, A\&A, 479, L37

Martins, F., Hillier, D. J., Paumard, T., et al. 2008, A\&A, 478, 219

Martins, F., Hillier, D. J., Bouret, J. C., et al. 2009, A\&A, 495, 257

Meynet, G., \& Maeder, A. 2005, A\&A, 429, 581

Meynet, G., Ekström, S., \& Maeder, A. 2006, A\&A, 447, 623

Müller, P. E., \& Vink, J. S. 2008, A\&A, 492, 493

Najarro, F., Figer, D. F., Hillier, D. J., \& Kudritzki, R. P. 2004, ApJ, 611, L105

Najarro, F., Figer, D. F., Hillier, D. J., Geballe, T. R., \& Kudritzki, R. P. 2009, ApJ, 691, 1816
Nugis, T., \& Lamers, H. J. G. L. M. 2000, A\&A, 360, 227

Nugis, T., \& Lamers, H. J. G. L. M. 2002, A\&A, 389, 162

Owocki, S., \& Shaviv, N. 2011, in Eta Carinae and the supernova imposters, ed. R. Humphreys, \& K. Davidson (Springer), in press

Pelupessy, I., Lamers, H. J. G. L. M., \& Vink, J. S. 2000, A\&A, 359, 695

Pereira, T. M. D., Asplund, M., \& Kiselman, D. 2009, A\&A, 508, 1403

Petrovic, J., Pols, O., \& Langer, N. 2006, A\&A, 450, 219

Pistinner, S., \& Eichler, D. 1995, ApJ, 454, 404

Portegies Zwart, S. F., Makino, J., McMillan, S. L. W., \& Hut, P. 1999, A\&A, 348,117

Prinja, R. K., \& Massa, D. L. 1998, in Properties of Hot Luminous Stars, ed. I. Howarth (San Francisco: ASP), ASP Conf. Ser., 131, 218

Ramírez, S. V., Sellgren, K., Carr, J. S., et al. 2000, ApJ, 537, 205

Rauw, G., Vreux, J.-M., Gosset, E., et al. 1996, A\&A, 306, 771

Rauw, G., De Becker, M., Nazé, Y., et al. 2004, A\&A, 420, L9

Reid, M. J. 1993, ARA\&A, 31, 345

Schaerer, D. 1996, A\&A, 309, 129

Schnurr, O., Casoli, J., Chené, A., Moffat, A. F. J., \& St-Louis, N. 2008, MNRAS, 389, L38

Schnurr, O., Moffat, A. F. J., Villar-Sbaffi, A., St-Louis, N., \& Morrell, N. I. 2009, MNRAS, 395, 823

Schweickhardt, J., Schmutz, W., Stahl, O., Szeifert, T., \& Wolf, B. 1999, A\&A, 347,127

Stolte, A., Grebel, E. K., Brandner, W., \& Figer, D. F. 2002, A\&A, 394, 459

Vink, J. S. 2006, in Stellar Evolution at Low Metallicity: Mass Loss, Explosions, Cosmology, ed. H. J. G. L. M. Lamers, N. Langer, T. Nugis, \& K. Annuk (San Francisco: ASP), ASP Conf. Ser., 353, 113

Vink, J. S., \& de Koter, A. 2002, A\&A, 393, 543

Vink, J. S., \& de Koter, A. 2005, A\&A, 442, 587

Vink, J. S., Muijres, L., Anthonisse, B., et al. 2011, A\&A, 531, A132

Woosley, S., \& Heger, A. 2006, ApJ, 637, 914

Yoon, S.-C., \& Langer, N. 2005, A\&A, 443, 643

Yoon, S., Langer, N., \& Norman, C. 2006, A\&A, 460, 199

Yungelson, L. R., van den Heuvel, E. P. J., Vink, J. S., Portegies Zwart, S. F., \& de Koter, A. 2008, A\&A, 477, 223 